\title{
HANKEL TENSOR DECOMPOSITIONS AND RANKS
}

\author{
JIAWANG NIE AND KE YE
}

\begin{abstract}
Hankel tensors are generalizations of Hankel matrices. This article studies both the computational and algebraic aspects of Hankel tensor ranks. We prove that for a low rank symmetric tensor, there exists a base change to make it a Hankel tensor. We also provide an algorithm that can compute the Vandermonde ranks and decompositions for all Hankel tensors. For a generic $n$-dimensional Hankel tensor of even order or order three, we prove that the candecomp-parafac rank, symmetric rank, Vandermonde rank, border rank, symmetric border rank, and Vandermonde border rank all coincide with each other. Some open questions are also posed.
\end{abstract}

\section{INTRODUCTION}

1.1. Various ranks for tensors. For integers $m, n>0$, denote by $\mathrm{T}^{m}\left(\mathbb{C}^{n}\right)$ the space of all $n$-dimensional complex tensors of order $m$. A tensor $\mathcal{A} \in \mathrm{T}^{m}\left(\mathbb{C}^{n}\right)$ is an array indexed by an integer tuple $\left(i_{1}, \ldots, i_{m}\right)$ in the range $1 \leq i_{1}, \ldots, i_{m} \leq n$, that is,

$$
\mathcal{A}=\left(\mathcal{A}_{i_{1} \ldots i_{m}}\right)_{1 \leq i_{1}, \ldots, i_{m} \leq n} .
$$

The tensor $\mathcal{A}$ is symmetric if $\mathcal{A}_{i_{1} \ldots i_{m}}$ is invariant with respect to all permutations of $\left(i_{1}, \ldots, i_{m}\right)$. Denote by $\mathrm{S}^{m}\left(\mathbb{C}^{n}\right)$ the vector space of all $n$-dimensional complex symmetric tensors of order $m$.

There are various types of ranks for tensors, which measure the complexity of tensor computations from different aspects. The typical ones are the candecomp-parafac (cp) rank [27] (also called just simply the rank, or the canonical polyadic rank, in some references), multilinear rank 14], tensor network rank [61, and nuclear rank 20, 40, (the nuclear rank of a tensor is defined to be the smallest length of its decompositions that achieve the nuclear norm). The symmetric rank 12 is defined for symmetric tensors. The Vandermonde rank [54] is defined for Hankel tensors. The border rank for general tensors and symmetric border rank for symmetric tensors are also defined for studying algebraic properties. We refer to 35, 41 for various definitions of tensor ranks. For convenience of reading, we shortly review them in the sequel.

All tensors can be expressed as linear combinations of outer products of vectors. For $u_{1}, \ldots, u_{m} \in \mathbb{C}^{n}$, the outer product $u_{1} \otimes \cdots \otimes u_{m}$ is the tensor in $\mathrm{T}^{m}\left(\mathbb{C}^{n}\right)$ such that for all $1 \leq i_{1}, \ldots, i_{m} \leq n$

$$
\left(u_{1} \otimes \cdots \otimes u_{m}\right)_{i_{1} \ldots i_{m}}=\left(u_{1}\right)_{i_{1}} \cdots\left(u_{m}\right)_{i_{m}} .
$$

The tensors of the form $u_{1} \otimes \cdots \otimes u_{m}$ are called rank-1 tensors. The $c p$ rank of $\mathcal{A} \in \mathrm{T}^{m}\left(\mathbb{C}^{n}\right)$ is defined as

$$
\operatorname{rank}(\mathcal{A}):=\min \left\{r \in \mathbb{N}: \mathcal{A}=\sum_{i=1}^{r} u_{i, 1} \otimes \cdots \otimes u_{i, m}, u_{i, j} \in \mathbb{C}^{n}\right\} .
$$

If $\operatorname{rank}(\mathcal{A})=r$, the corresponding decomposition in (1.1) is called a rank decomposition of $\mathcal{A}$. The border rank of $\mathcal{A}$ is then defined as

$$
\operatorname{brank}(\mathcal{A}):=\min \left\{r \in \mathbb{N}: \mathcal{A}=\lim _{p \rightarrow \infty} \sum_{i=1}^{r} u_{i, 1}^{(p)} \otimes \cdots \otimes u_{i, m}^{(p)}, u_{i, j}^{(p)} \in \mathbb{C}^{n}\right\} .
$$

Clearly, it always holds that $\operatorname{brank}(\mathcal{A}) \leq \operatorname{rank}(\mathcal{A})$.

For symmetric tensors, we are typically interested in their symmetric ranks. For $\mathcal{A} \in \mathrm{S}^{m}\left(\mathbb{C}^{n}\right)$, its symmetric rank is defined as

$$
\operatorname{rank}_{S}(\mathcal{A}):=\min \left\{r \in \mathbb{N}: \mathcal{A}=\sum_{i=1}^{r}\left(u_{i}\right)^{\otimes m}, u_{i} \in \mathbb{C}^{n}\right\} .
$$

2010 Mathematics Subject Classification. 15A18, 15A69.

Key words and phrases. Hankel tensor, rank, Vandermonde decomposition, Waring decomposition. 
In the above, $\left(u_{i}\right)^{\otimes m}:=u_{i} \otimes \cdots \otimes u_{i}$, where $u_{i}$ is repeated $m$ times. If $\operatorname{rank}_{S}(\mathcal{A})=r$, the corresponding decomposition in (1.2) is called a symmetric rank decomposition of $\mathcal{A}$. A symmetric tensor $\mathcal{A} \in \mathrm{S}^{m}\left(\mathbb{C}^{n}\right)$ can be uniquely represented by a homogeneous polynomial (i.e., a form) of degree $m$ and in $\left(x_{1}, \ldots, x_{n}\right)$, which is

$$
\mathcal{A}(x):=\sum_{1 \leq i_{1}, \ldots, i_{m} \leq n} \mathcal{A}_{i_{1} \cdots i_{m}} x_{i_{1}} \cdots x_{i_{m}} .
$$

A symmetric decomposition of $\mathcal{A}$ is equivalent to the decomposition of $\mathcal{A}(x)$ as a sum of powers of linear forms. In the literature, the symmetric rank decomposition is also called a Waring decomposition, and the symmetric rank is also called Waring rank [35, 48. The symmetric rank of a form means the symmetric rank of the corresponding symmetric tensor. The symmetric border rank of $\mathcal{A} \in \mathrm{S}^{m}\left(\mathbb{C}^{n}\right)$ is then defined as

$$
\operatorname{brank}_{S}(\mathcal{A}):=\min \left\{r: \lim _{p \rightarrow \infty} \sum_{i=1}^{r} u_{i}^{(p)}{ }^{\otimes m}=\mathcal{A}, u_{i}^{(p)} \in \mathbb{C}^{n}\right\} .
$$

For a symmetric tensor $\mathcal{A}$, it is straightforward to see that

$$
\operatorname{brank}(\mathcal{A}) \leq \operatorname{brank}_{S}(\mathcal{A}) \leq \operatorname{rank}_{S}(\mathcal{A})
$$

Determining ranks and decompositions of tensors are fundamental questions in many applications, such as signal processing [11, 40, multiway factor analysis [14, and computational complexity 6, 60]. A general survey about applications can be found in [30. It is NP-hard to compute the most ranks 1 and decompositions of tensors [25, 26]. Even for symmetric tensors, the question of computing their symmetric ranks and Waring decompositions still remains NP-hard [55. We refer to the work [3, 15, 18, 38, for general tensor decompositions and refer to the work [4, 5, 44, 48, for symmetric tensor decompositions. Other interesting questions about tensors include low rank approximations [16, 22, 29, 43, 45, uniqueness of tensor decompositions [9, 21, 31, symmetric rank of monomials 34, 49, defining ideals of low rank tensors [32, 33, and tensor eigenvalues [13, 39, 46, 52, 53.

1.2. Hankel tensors. A tensor $\mathcal{H} \in \mathrm{T}^{m}\left(\mathbb{C}^{n}\right)$ is called Hankel if $\mathcal{H}_{i_{1} \ldots i_{m}}$ is invariant whenever the sum $i_{i}+\cdots+i_{m}$ is a constant [51, 54]. In other words, $\mathcal{H}$ is a Hankel tensor if and only if there exists a vector $h:=\left(h_{0}, h_{1}, \ldots, h_{(n-1) m}\right)$ such that

$$
\mathcal{H}_{i_{1} \ldots i_{m}}=h_{i_{1}+\cdots+i_{m}-m} .
$$

Clearly, each Hankel tensor is also symmetric. We denote by $\mathrm{H}^{m}\left(\mathbb{C}^{n}\right)$ the linear subspace of all Hankel tensors in $\mathrm{S}^{m}\left(\mathbb{C}^{n}\right)$. It is easy to obtain that the dimension of $\mathrm{H}^{m}\left(\mathbb{C}^{n}\right)$ is $(n-1) m+1$. As shown by Qi [54, $\mathcal{H}$ is a Hankel tensor if and only if it has a Vandermonde decomposition, i.e., for some $\lambda_{i}, t_{i} \in \mathbb{C}$,

$$
\mathcal{H}=\sum_{i=1}^{r} \lambda_{i}\left(1, t_{i}, \ldots, t_{i}^{n-1}\right)^{\otimes m}
$$

In this paper, we consider the homogenization of the above:

$$
\mathcal{H}=\sum_{i=1}^{r}\left(a_{i}^{n-1}, a_{i}^{n-2} b_{i}, \ldots, a_{i} b_{i}^{n-2}, b_{i}^{n-1}\right)^{\otimes m} .
$$

Definition 1.1. For a Hankel tensor $\mathcal{H}$, the smallest integer $r$ for (1.4) to hold is called the Vandermonde rank (or just $V$-rank) of $\mathcal{H}$, which is denoted by $\operatorname{rank}_{V}(\mathcal{H}$ ). The corresponding decomposition is called the Vandermonde rank decomposition or just the $V$-rank decomposition. The Vandermonde border rank (or just the border $V$-rank) of $\mathcal{H}$, which we denote as $\operatorname{brank}_{V}(\mathcal{H})$, is the smallest $r$ such that $\mathcal{H}$ is the limit of a sequence of Hankel tensors whose $V$-rank is $r$.

We remark that the simplest $n$-dimensional Hankel tensor of order $m$ is of the form

$$
\left(a^{n-1}, a^{n-2} b, \ldots, a b^{n-2}, b^{n-1}\right)^{\otimes m}, \quad a, b \in \mathbb{C}
$$

and such a tensor has the Vandermonde rank one. If we consider $\mathcal{H} \in \mathrm{H}^{m}\left(\mathbb{C}^{n}\right)$ as a tensor in $\mathrm{T}^{m}\left(\mathbb{C}^{n}\right)$, its cp $\operatorname{rank} \operatorname{rank}(\mathcal{H})$ is defined in (1.1); if we consider it as a tensor in $\mathrm{S}^{m}\left(\mathbb{C}^{n}\right)$, its symmetric $\operatorname{rank} \operatorname{rank}_{S}(\mathcal{H})$ is

\footnotetext{
1 There exist some ranks and decompositions which are easy to compute, for instance, multi-linear rank and higher order singular value decomposition (HOSVD).
} 
defined in (1.2). The border $\operatorname{rank} \operatorname{brank}(\mathcal{H})$ and symmetric border $\operatorname{rank} \operatorname{brank}_{S}(\mathcal{H})$ are defined in the same way.

For a Hankel tensor $\mathcal{H}$, it clearly holds that

$$
\operatorname{brank}(\mathcal{H}) \leq \operatorname{rank}(\mathcal{H}) \leq \operatorname{rank}_{S}(\mathcal{H}) \leq \operatorname{rank}_{V}(\mathcal{H}) .
$$

For the relations among various ranks of a Hankel tensor $\mathcal{H}$, we have the following two simple facts:

1) The $V$-rank of $\mathcal{H}$ is 1 if and only if $\operatorname{rank}_{S}(\mathcal{H})=1$. Clearly, if $\operatorname{rank}_{V}(\mathcal{H})=1$, then $\mathcal{H} \neq 0$ and hence $\operatorname{rank}_{S}(\mathcal{H}) \geq 1$, so they are the same by (1.5). Conversely, if $\operatorname{rank}_{S}(\mathcal{H})=1$, then $\mathcal{H}=v^{\otimes m}$, for some $v=\left(v_{1}, \ldots, v_{n}\right) \in \mathbb{C}^{n}$. Since $\mathcal{H}$ is Hankel, $v_{i_{1}} \cdots v_{i_{m}}=v_{j_{1}} \cdots v_{j_{m}}$ for all $i_{1}+\cdots+i_{m}=j_{1}+\cdots+j_{m}$. In particular, for $i_{1}=\cdots=i_{m}=i$ and $j_{1}=\cdots=j_{m-2}=i, j_{m-1}=i-1, j_{m}=i+1$, we get $v_{i-1} v_{i+1}=v_{i}^{2}$ for $i=2, \ldots, n-1$. Therefore, we can parametrize $v$ as $v=\left(a^{n-1}, a^{n-2} b, \ldots, b^{n-1}\right)$, so $\operatorname{rank}_{V}(\mathcal{H})=1$. For this case, all the ranks are the same by (1.5).

2) For the binary case (i.e., $n=2$ ), we also have $\operatorname{rank}_{S}(\mathcal{H})=\operatorname{rank}_{V}(\mathcal{H})$. This is because for every 2-dimensional vector $v=(a, b)$, the tensor power $v^{\otimes m}$ is itself a Vandermonde decomposition. When $n=2$, it holds that

$$
\mathrm{H}^{m}\left(\mathbb{C}^{2}\right)=\mathrm{S}^{m}\left(\mathbb{C}^{2}\right) \simeq \mathbb{C}^{m+1}
$$

Hankel tensors have broad applications. They were originally defined in signal processing [51] for studying the Harmonic Retrieval problem 42. Moreover, Hankel tensors can also be used to solve the interpolation problem [59]. Recently, Qi 54] studied Vandermonde decompositions and complete/strong Hankel tensors. The inheritance properties and sum-of-squares decompositions for Hankel tensors are studied by Ding, Qi and Wei [17. Extremal eigenvalues of Hankel tensors are discussed in Chen, Qi and Wang [8]. Some further results about Hankel tensors appear in Chen, Li and Qi [7].

1.3. Contributions. The $V$-rank decompositions of Hankel tensors are closely related to symmetric rank decompositions of binary forms. Let $d:=(n-1) m$ and let $h$ be as in (1.3). The vector $h$ can be uniquely identified as a binary form of degree $d$ :

$$
h(x, y):=\sum_{j=0}^{d}\left(\begin{array}{l}
d \\
j
\end{array}\right) h_{j} x^{j} y^{d-j} .
$$

It can also be thought of as a symmetric binary tensor of order $d$. By writing $\operatorname{rank}_{S}(h)\left(\operatorname{resp}\right.$., brank $\left.k_{S}(h)\right)$, we mean the symmetric rank (resp., symmetric border rank) when $h$ is regarded as the symmetric tensor represented by the binary form $h(x, y)$. Note that $\operatorname{rank}_{S}(h)$ is just the Waring rank of the form $h(x, y)$. The Vandermonde decomposition (1.4) is equivalent to

$$
h(x, y)=\sum_{i=1}^{r}\left(a_{i} x+b_{i} y\right)^{d} .
$$

The symmetric rank of $h$ is the smallest $r$ in the above. In Lemma 3.2. we will show that $\operatorname{rank}_{V}(\mathcal{H})=$ $\operatorname{rank}_{S}(h)$.

This article focuses on various ranks of Hankel tensors. We mainly address the following two basic questions:

- How can we determine the Vandermonde rank and decomposition of a Hankel tensor?

- What are the relations among various ranks of a Hankel tensor?

First, we propose an algorithm (Algorithm 3.4) that can compute the Vandermonde rank and decomposition for all Hankel tensors. This will be done in Section 3

Second, we show that the cp rank, symmetric rank, border rank, symmetric border rank and Vandermonde rank are the same for a generic $\mathcal{H} \in \mathrm{H}^{m}\left(\mathbb{C}^{n}\right)$ when $m$ is even or $m=3$. In particular, this implies that Comon's conjecture 47 is true for generic Hankel tensors of even order or order three. Moreover, for a specifically given Hankel tensor, we give concrete conditions for determining these ranks. This will be done in Sections 4 and 5

We give some preliminary results in Section 2, and conclude the paper with some open questions and conjectures in Section 6 . 


\section{Preliminaries}

Notation. The symbol $\mathbb{N}$ (resp., $\mathbb{R}, \mathbb{C}$ ) denotes the set of nonnegative integers (resp., real, complex numbers). The symbol $\mathbb{C}[x]:=\mathbb{C}\left[x_{1}, \ldots, x_{n}\right]$ denotes the ring of polynomials in $x:=\left(x_{1}, \ldots, x_{n}\right)$ over the complex field $\mathbb{C}$. For any $t \in \mathbb{R},\lceil t\rceil$ (resp., $\lfloor t\rfloor$ ) denotes the smallest integer not smaller (resp., the largest integer not bigger) than $t$. The cardinality of a set $S$ is denoted as $\# S$. For a matrix $M$, its null space is denoted as $\operatorname{ker} M$.

2.1. Elementary algebraic geometry. For basics in algebraic geometry, we refer to $\left[24\right.$. A set $X \subseteq \mathbb{C}^{n}$ is an algebraic variety if there exist polynomials $f_{1}, \ldots, f_{s} \in \mathbb{C}[x]$ such that

$$
X=\left\{a \in \mathbb{C}^{n}: f_{1}(a)=\cdots=f_{s}(a)=0\right\} .
$$

The Zariski topology on $\mathbb{C}^{n}$ is the topology such that the closed sets are algebraic varieties. An algebraic variety is called irreducible if it is not a union of two proper algebraic varieties. We need the notion of a generic point in an irreducible algebraic variety $V$. For a property $P$ on $V$, we say that a generic point in $V$ has the property $P$ if the set of points in $V$ which do not satisfy $P$ is contained in a proper closed subset of $V$ in the Zariski topology. For instance, a generic point $(x, y) \in \mathbb{C}^{n} \times \mathbb{C}^{n}$ uniquely determines a line $L \subset \mathbb{C}^{n}$ such that $x, y \in L$. This is because lines passing through $x, y$ are not unique if and only if $x=y$ and the set $\left\{(x, y) \in \mathbb{C}^{n} \times \mathbb{C}^{n}: x=y\right\}$ is a proper closed subset of $\mathbb{C}^{n} \times \mathbb{C}^{n}$. If the property $P$ is clear from the context, we just say "a generic point" without mentioning $P$.

The projective space $\mathbb{P}^{n}$ consists of all lines in $\mathbb{C}^{n+1}$, or equivalently, $\mathbb{P}^{n}$ is the set of equivalence classes

$$
\left[\left(a_{0}, \ldots, a_{n}\right)\right]=\left\{\left(\lambda a_{0}, \ldots, \lambda a_{n}\right) \in \mathbb{C}^{n+1}:\left(a_{0}, \ldots, a_{n}\right) \neq 0, \lambda \neq 0\right\} .
$$

A subset $X \subseteq \mathbb{P}^{n}$ is a projective variety if there exist homogeneous polynomials $f_{1}, \ldots, f_{s} \in \mathbb{C}\left[x_{0}, \ldots, x_{n}\right]$ such that

$$
[a] \in X \text { if and only if } f_{1}(a)=\cdots=f_{s}(a)=0 .
$$

2.2. Multilinear algebra. Let $B$ be a vector space of dimension $n$ and let $\left\{b_{1}, \ldots, b_{n}\right\}$ be a set of basis. For an integer $1 \leq p \leq n$, the $p$-th exterior power of $B$, denoted as $\wedge^{p} B$, is the vector space spanned by the $\left(\begin{array}{l}n \\ p\end{array}\right)$ vectors $b_{j_{1}} \wedge \cdots \wedge b_{j_{p}}\left(1 \leq j_{1}<\cdots<j_{p} \leq n\right)$, where the wedge product $v_{1} \wedge \cdots \wedge v_{p}$ is defined as

$$
v_{1} \wedge \cdots \wedge v_{p}:=\sum_{\sigma \in \mathfrak{S}_{p}} \operatorname{sgn}(\sigma) v_{\sigma(1)} \otimes \cdots \otimes v_{\sigma(p)} .
$$

In the above, $\mathfrak{S}_{p}$ is the permutation group on $p$ elements and $\operatorname{sgn}(\sigma)$ is the sign of the permutation $\sigma \in \mathfrak{S}_{p}$. In particular, we have

$$
v \wedge v=0, \quad v \in B
$$

Clearly, $\bigwedge^{p} B$ is a linear subspace of $B^{\otimes n}$, with dimension $\left(\begin{array}{l}n \\ p\end{array}\right)$. Moreover,

$$
v_{1} \wedge \cdots \wedge v_{p}=\operatorname{sgn}(\sigma) v_{\sigma(1)} \wedge \cdots \wedge v_{\sigma(p)}, \quad \forall \sigma \in \mathfrak{S}_{p}
$$

The exterior power $\bigwedge^{p} B$ is a generalization of skew-symmetric matrices. Indeed, if $p=2$, then $\bigwedge^{2} B$ is simply the vector space of all $n \times n$ skew symmetric matrices.

Let $A, B, C$ be vector spaces of dimensions $m, n, q$ respectively. Every tensor $T \in A \otimes B \otimes C$ can be regarded as a linear map $\varphi_{T}: A^{*} \rightarrow B \otimes C$. If we choose bases $\left\{a_{1}, \ldots, a_{m}\right\},\left\{b_{1}, \ldots, b_{n}\right\}$ and $\left\{c_{1}, \ldots, c_{q}\right\}$ for $A, B, C$ respectively, then we can write $T$ as

$$
T=\sum_{i=1}^{m} \sum_{j=1}^{n} \sum_{k=1}^{q} t_{i j k} a_{i} \otimes b_{j} \otimes c_{k} .
$$

Let $\left\{\alpha_{1}, \ldots, \alpha_{m}\right\}$ be the dual basis of $A^{*}$, then $\varphi_{T}$ is given by

$$
\varphi_{T}\left(\alpha_{i}\right)=\sum_{j=1}^{n} \sum_{k=1}^{q} t_{i j k} b_{j} \otimes c_{k} .
$$

For each integer $1 \leq p \leq n-1$, define $\varphi_{T}^{p}$ to be the linear map

$$
\varphi_{T}^{p}:\left(\bigwedge^{p} B\right) \otimes A^{*} \rightarrow\left(\bigwedge^{p+1} B\right) \otimes C
$$


which is obtained by tensoring $\varphi_{T}$ with the identity map $\operatorname{Id}_{\wedge^{p} B}: \bigwedge^{p} B \rightarrow \bigwedge^{p} B$ and projecting the image of $\varphi_{T} \otimes \operatorname{Id}_{\bigwedge^{p} B}$ in $\left(\bigwedge^{p} B\right) \otimes B \otimes C$ onto $\left(\bigwedge^{p+1} B\right) \otimes C$. Here, the projection of $\bigwedge^{p} B \otimes B \otimes C$ onto $\bigwedge^{p+1} B \otimes C$ is determined by

$$
\left(b_{1} \wedge \cdots \wedge b_{p}\right) \otimes b_{p+1} \otimes c \mapsto\left(b_{1} \wedge \cdots \wedge b_{p} \wedge b_{p+1}\right) \otimes c .
$$

To be more specific, the linear map $\varphi_{T}^{p}$ is defined such that

$$
\left(b_{1} \wedge \cdots \wedge b_{p}\right) \otimes \alpha_{i} \mapsto \sum_{j=1}^{n} \sum_{k=1}^{q} t_{i j k}\left(b_{1} \wedge \cdots \wedge b_{p} \wedge b_{j}\right) \otimes c_{k}
$$

Theorem 2.1. [37, Theorem 2.1] Let $T$ be a tensor in $A \otimes B \otimes C$ and $0<p<n$ be an integer. If $\varphi_{T}^{p}$ is the linear map defined in (2.1), then

$$
\operatorname{brank}(T) \geq \operatorname{rank} \varphi_{T}^{p} /\left(\begin{array}{c}
n-1 \\
p
\end{array}\right) .
$$

Theorem 2.1 can be used to obtain Strassen equations for bounded border rank tensors, see [35, p. 81] and 50, Theorem 3.2]. For instance, when $\operatorname{dim} A=\operatorname{dim} B=\operatorname{dim} C=2$ and $p=1$, a tensor $T \in A \otimes B \otimes C$ can be written as $T=\sum_{i, j, k=1}^{2} t_{i j k} a_{i} \otimes b_{j} \otimes c_{k}$. Hence, $\varphi_{T}\left(\alpha_{i}\right)=\sum_{j, k=1}^{2,2} t_{i j k} b_{j} \otimes c_{k}$. This implies that $\varphi_{T}^{p}: B \otimes A^{*} \rightarrow \bigwedge^{2} B \otimes C$ is defined as

$$
\varphi_{T}^{p}\left(b \otimes \alpha_{i}\right)=\sum_{j, k=1}^{2,2} t_{i j k}\left(b_{j} \wedge b\right) \otimes c_{k} .
$$

It corresponds to the $4 \times 2$ matrix

$$
M_{T}^{p}:=\begin{gathered}
b_{1} \otimes \alpha_{1} \\
b_{1} \otimes \alpha_{2} \\
b_{2} \otimes \alpha_{1} \\
b_{2} \otimes \alpha_{2}
\end{gathered}\left[\begin{array}{cc}
\left(b_{1} \wedge b_{2}\right) \otimes c_{1} & \left(b_{1} \wedge b_{2}\right) \otimes c_{2} \\
-t_{121} & -t_{122} \\
-t_{221} & -t_{222} \\
t_{111} & t_{112} \\
t_{211} & t_{212}
\end{array}\right]
$$

The rows of $M_{T}^{p}$ are indexed by the basis vectors $b_{1} \otimes \alpha_{1}, b_{1} \otimes \alpha_{2}, b_{2} \otimes \alpha_{1}, b_{2} \otimes \alpha_{2}$ of $B \otimes A^{*}$ and whose columns are indexed by the basis vectors $\left(b_{1} \wedge b_{2}\right) \otimes c_{1},\left(b_{1} \wedge b_{2}\right) \otimes c_{2}$ of $\left(\wedge^{2} B\right) \otimes C$. For readers' convenience, we label rows and columns with their corresponding bases, respectively. In the literature, the linear map $\varphi_{T}^{p}$ defined as in (2.1) is called a Young flattening or a Koszul flattening of the tensor T. We refer to [35, p. 81] and [48, Example 4.2].

2.3. Symmetric ranks of binary forms. A binary form $h(x, y)$ is a homogeneous polynomial in two variables $x, y$. Every binary form of degree $d$ can be regarded as a symmetric binary tensor of order $d$, so $S^{d}\left(\mathbb{C}^{2}\right) \simeq \mathbb{C}^{d+1}$. The symmetric rank of the symmetric tensor represented by $h(x, y)$ is also called the symmetric rank of $h(x, y)$. We can write $h(x, y)=\sum_{i=0}^{d}\left(\begin{array}{c}d \\ i\end{array}\right) h_{i} x^{i} y^{d-i}$. For convenience, we denote

$$
h:=\left(h_{0}, h_{1}, \ldots, h_{d}\right) .
$$

For $0 \leq r \leq d$, we denote the Hankel matrix

$$
C_{d-r, r}(h):=\left[\begin{array}{cccc}
h_{0} & h_{1} & \cdots & h_{r} \\
h_{1} & h_{2} & \cdots & h_{r+1} \\
\vdots & \vdots & \ddots & \vdots \\
h_{d-r} & h_{d-r+1} & \cdots & h_{d}
\end{array}\right] \in \mathbb{C}^{(d-r+1) \times(r+1)} .
$$

The symmetric rank $\operatorname{rank}_{S}(h)$ of $h(x, y)$ can be determined as follows.

Theorem 2.2. 10, Theorem 11] Let $h(x, y)=\sum_{i=0}^{d} h_{i}\left(\begin{array}{l}d \\ i\end{array}\right) x^{i} y^{d-i}$ be a binary form of degree $d$. Then, we have the following:

- The symmetric border rank of $h$ is

$$
r:=\operatorname{rank} C_{\left\lceil\frac{d}{2}\right\rceil,\left\lfloor\frac{d}{2}\right\rfloor}(h) .
$$

- If $d$ is even and $r=d / 2+1$, then $\operatorname{rank}_{S}(h)=r$. 
- If $d$ is odd, or if $r<d / 2+1$, then $\operatorname{rank}_{S}(h)=r$ or $d-r+2$ which can be determined as follows: let $\left(f_{0}, f_{1}, \ldots, f_{r}\right) \neq 0$ be a vector from $\operatorname{ker} C_{d-r, r}(h)$, which is unique up to scaling. If the polynomial

$$
f(x, y):=f_{0} x^{r}+f_{1} x^{r-1} y+\cdots+f_{r} y^{r}
$$

has no multiple roots, then $\operatorname{rank}_{S}(h)=r$; otherwise, $\operatorname{rank}_{S}(h)=d-r+2$.

Once we know the symmetric rank $k:=\operatorname{rank}_{S}(h)$, Sylvester's method can be applied to compute the Waring decomposition of the binary form $h(x, y)$. By the above theorem, either $k=r$ or $k=d-r+2$. Select a generic vector $0 \neq\left(g_{0}, g_{1}, \ldots, g_{k}\right) \in \operatorname{ker} C_{d-k, k}(h)$. Then, the binary form

$$
g(x, y):=g_{0} x^{k}+g_{1} x^{k-1} y+\cdots+g_{k} y^{k}
$$

has $k$ distinct complex roots, say, $\left(a_{1}, b_{1}\right), \ldots,\left(a_{k}, b_{k}\right)$, in the projective space $\mathbb{P}^{1}$. Moreover, there exist scalars $\lambda_{1}, \ldots, \lambda_{k}$ satisfying

$$
h(x, y)=\lambda_{1}\left(a_{1} x+b_{1} y\right)^{d}+\cdots+\lambda_{k}\left(a_{k} x+b_{k} y\right)^{d} .
$$

The above is justified by the following theorem of Sylvester.

Theorem 2.3 ([57, [58]). A binary form $h(x, y)=\sum_{i=0}^{d} h_{i}\left(\begin{array}{c}d \\ i\end{array}\right) x^{i} y^{d-i}$ of degree $d$ has the decomposition $h(x, y)=\sum_{i=1}^{k} \lambda_{i}\left(a_{i} x+b_{i} y\right)^{d}$ for $\lambda_{1}, \ldots, \lambda_{k} \neq 0$ and $\left(a_{1}, b_{1}\right), \ldots,\left(a_{k}, b_{k}\right) \in \mathbb{C}^{2}$ pairwise linearly independent if and only if there exists $0 \neq\left(g_{0}, g_{1}, \ldots, g_{k}\right) \in \operatorname{ker} C_{d-k, k}(h)$ such that the binary form $g(x, y)$ as in (2.4) has $\left(a_{1}, b_{1}\right), \ldots,\left(a_{k}, b_{k}\right)$ as complex roots in $\mathbb{P}^{1}$.

\section{VANDERMONDE RANKS AND DECOMPOSITIONS}

Although Hankel tensors are seemly very special symmetric tensors, every low rank symmetric tensor can be written as a Hankel tensor under a change of coordinate. More precisely, we have the following:

Proposition 3.1. For a generic symmetric tensor $\mathcal{S} \in \mathrm{S}^{m}\left(\mathbb{C}^{n}\right)$ such that $\operatorname{rank}_{S}(\mathcal{S}) \leq n+2$, there exists an $n \times n$ invertible matrix $G$ such that

$$
G \cdot \mathcal{S} \in \mathrm{H}^{m}\left(\mathbb{C}^{n}\right) \text { and } \operatorname{rank}_{S}(\mathcal{S})=\operatorname{rank}_{V}(G \cdot \mathcal{S}),
$$

where $\cdot$ is the diagonal action 2 In particular, if $\mathcal{H} \in \mathrm{H}^{m}\left(\mathbb{C}^{n}\right)$ is generi ${ }^{3}$ such that $\operatorname{rank}_{S}(\mathcal{H}) \leq n+2$, then we must have $\operatorname{rank}_{V}(\mathcal{H})=\operatorname{rank}_{S}(\mathcal{H})$.

Proof. Since $\mathcal{S}$ has symmetric rank at most $(n+2)$, we can write $\mathcal{S}$ as

$$
\mathcal{S}=\sum_{j=1}^{r} u_{j}^{\otimes m}, \quad r \leq n+2 .
$$

Since $\mathcal{S}$ is generic, we may assume that $u_{1}, \ldots, u_{r}$ are in general position, i.e., any $k$ of them span a linear subspace of dimension $\min \{k, n\}$ for each $k=1, \ldots, r$. By [24, Theorem 1.18], there is a unique curve $C \subset \mathbb{P}^{n-1}$ passing through $\left[u_{1}\right], \ldots,\left[u_{n+2}\right] \in \mathbb{P}^{n-1}$, which is projectively equivalent to the rational normal curve $v_{n}\left(\mathbb{P}^{1}\right)$. Therefore there exists an $n \times n$ invertible matrix $G$ such that

$$
G u_{j}=\left(a_{j}^{n-1}, a_{j}^{n-2} b_{j}, \ldots, a_{j} b_{j}^{n-2}, b_{j}^{n-1}\right), \quad\left(a_{j}, b_{j}\right) \in \mathbb{C}^{2}, \quad 1 \leq j \leq n+2
$$

and hence $G \cdot \mathcal{S} \in \mathrm{H}^{m}\left(\mathbb{C}^{n}\right)$. The second part follows easily from the first part.

For a Hankel tensor $\mathcal{H} \in \mathrm{H}^{m}\left(\mathbb{C}^{n}\right)$, let $d=(n-1) m$ and $h=\left(h_{0}, h_{1}, \ldots, h_{d}\right)$ be the vector as in (1.3). We can think of $h$ as the symmetric binary tensor in $\mathrm{S}^{d}\left(\mathbb{C}^{2}\right)$ that is represented by the binary form

$$
h(x, y):=\sum_{i=0}^{d} h_{i}\left(\begin{array}{l}
d \\
i
\end{array}\right) x^{i} y^{d-i} .
$$

\footnotetext{
${ }^{2}$ The diagonal action $G \cdot \mathcal{S}$ is defined as: if $\mathcal{S}=\sum_{i}\left(u_{i}\right)^{\otimes m}$ is a decomposition, then $G \cdot \mathcal{S}=\sum{ }_{i}\left(G u_{i}\right)^{\otimes m}$.

${ }^{3} \mathcal{H}$ is a generic element in the set of all Hankel tensors with $\operatorname{rank}_{S}(\mathcal{H}) \leq n+2$.

4 Two projective varieties $X, Y \subseteq \mathbb{P}^{n-1}$ are projectively equivalent if there exists an invertible $n \times n$ matrix $G$ such that $Y=G X$.
} 
By writing $\operatorname{rank}_{S}(h)$ (resp., brank ${ }_{S}(h)$ ), we mean the symmetric rank (resp., the symmetric border rank) of the tensor represented by $h(x, y)$. There is a one-to-one linear map between Hankel tensors and binary forms, which is given as:

$$
\pi: \mathrm{H}^{m}\left(\mathbb{C}^{n}\right) \rightarrow \mathrm{S}^{d}\left(\mathbb{C}^{2}\right), \quad \mathcal{H} \mapsto h .
$$

In the above, $h$ is determined by $\mathcal{H}$ as in (1.3). Clearly, the map $\pi$ is a bijection between $\mathrm{H}^{m}\left(\mathbb{C}^{n}\right)$ and $\mathrm{S}^{d}\left(\mathbb{C}^{2}\right)$, and $\operatorname{rank}_{S}(h)=1$ if and only if $\operatorname{rank}_{V}(\mathcal{H})=1$. A Vandermonde rank decomposition of $\mathcal{H}$ is equivalent to a symmetric rank decomposition of $h$. We have the following basic lemma.

Lemma 3.2. Let $\pi, \mathcal{H}, h$ be as above. Then, for all $\mathcal{H} \in \mathrm{H}^{m}\left(\mathbb{C}^{n}\right)$, it holds that

$$
\operatorname{rank}_{V}(\mathcal{H})=\operatorname{rank}_{S}(h), \quad \operatorname{brank}_{V}(\mathcal{H})=\operatorname{brank}_{S}(h) .
$$

Proof. We notice that $\pi$ is actually an isomorphism between two vector spaces. This implies in particular that $\pi$ is continuous if we equip both vector spaces the Euclidean topology. Hence it is sufficient to prove $\operatorname{rank}_{V}(\mathcal{H})=\operatorname{rank}_{S}(h)$ and the border version follows from the continuity of $\pi$. To this end, since $\pi$ is a bijective correspondence between rank one tensors, $\operatorname{rank}_{V}(\mathcal{H})=\operatorname{rank}_{S}(h)$ follows from the linearity of $\pi$.

Lemma 3.2 establishes the equivalence between Vandermonde decompositions of Hankel tensors and symmetric decompositions of binary forms. Therefore, all results about binary forms can be applied directly to Hankel tensors via the map $\pi$. When a Hankel tensor $\mathcal{H}$ is generic, the symmetric binary tensor $h$ is also generic. By Theorems 2.2, 2.3 and Lemma 3.2. we can get the following corollary about Vandermonde ranks of generic Hankel tensors.

Corollary 3.3. If $\mathcal{H} \in \mathrm{H}^{m}\left(\mathbb{C}^{n}\right)$ is generic, then

$$
\operatorname{rank}_{V}(\mathcal{H})=\left\lceil\frac{(n-1) m+1}{2}\right\rceil .
$$

The symmetric rank and the decomposition of a binary form is determined in Theorem 2.2. Consequently, by Lemma 3.2. we can determine the Vandermonde rank decomposition of a Hankel tensor by the following algorithm.

Algorithm 3.4. (Vandermonde rank decompositions for Hankel tensors, motivated from Sylvester's algorithm for binary tensors.) For a given $\mathcal{H} \in \mathrm{H}^{m}\left(\mathbb{C}^{n}\right)$, let $d=(n-1) m$ and $h$ be as in (1.3). Do the following:

Step 1: Let $s=\lfloor d / 2\rfloor$ and form the matrix $C_{d-s, s}(h)$ as in (2.2.).

Step 2: Let $r:=\operatorname{rank} C_{d-s, s}(h)$. Find $0 \neq\left(f_{0}, f_{1}, \ldots, f_{r}\right) \in \operatorname{ker} C_{d-r, r}(h)$. Set $f(x, y):=f_{0} x^{r}+f_{1} x^{r-1} y+$ $\cdots+f_{r} y^{r}$.

Step 3: If $r=d / 2+1$ or $f(x, y)$ has no multiple roots, then $\operatorname{rank}_{V}(\mathcal{H})=\operatorname{rank}_{S}(h)=r$; otherwise, $\operatorname{rank}_{V}(\mathcal{H})=\operatorname{rank}_{S}(h)=d-r+2$.

Step 4: Let $k:=\operatorname{rank}_{V}(\mathcal{H})$, which is either $r$ or $d-r+2$. If $k=r$, compute the $r$ distinct roots $\left(a_{1}, b_{1}\right), \ldots,\left(a_{r}, b_{r}\right)$ of the binary form $f(x, y)$. If $k=d-r+2$, select a generic $0 \neq\left(g_{0}, g_{1}, \ldots, g_{k}\right) \in$ ker $C_{d-k, k}$ and compute the $k$ distinct roots $\left(a_{1}, b_{1}\right), \ldots,\left(a_{k}, b_{k}\right)$ of the binary form $g(x, y):=$ $g_{0} x^{k}+g_{1} x^{k-1} y+\cdots+g_{k} y^{k}$.

Step 5: Determine the scalars $\lambda_{1}, \ldots, \lambda_{k}$ such that

$$
\mathcal{H}=\sum_{i=1}^{k} \lambda_{i}\left(a_{i}^{n-1}, a_{i}^{n-2}\left(-b_{i}\right), \ldots,\left(-b_{i}\right)^{n-1}\right)^{\otimes m} .
$$

The above is equivalent to $h(x, y)=\sum_{i=1}^{k} \lambda_{i}\left(a_{i} x+b_{i} y\right)^{d}$.

Algorithm 3.4 is mathematically equivalent to computing rank decompositions of binary symmetric tensors, through the map $\pi$ defined in (3.2) and Lemma 3.2. It is orginated from Sylvester's algorithm [57, 58] (also see [5, Algorithm 1.1]) for the case of simple roots and the results of Comas and Seuger [10 for the case of multiple roots (also see [4, Algorithms 1,2]). For symmetric tensors of generic rank or subgeneric rank, the uniqueness of the rank decompositions is studied in [9] and 21. By Theorems 2.2 and 2.3 and Lemma 3.2. we can get similar uniqueness results about Vandermonde rank decompositions. 
Theorem 3.5. Let $\mathcal{H} \in \mathrm{H}^{m}\left(\mathbb{C}^{n}\right)$ be a Hankel tensor. In Algorithm 3.4, if the rank of $C_{d-s, s}(h)$ is less than $d / 2+1$ and the binary form $f(x, y)$ has no multiple roots, then the Vandermonde rank decomposition of $\mathcal{H}$ is unique.

3.1. Some examples. In the following, we give examples to show how Algorithm 3.4 works. We would like to remark that

1) It is possible that $\operatorname{rank}_{V}(\mathcal{H})>\operatorname{rank}_{S}(\mathcal{H})$.

2) The symmetric rank decomposition of $\mathcal{H}$ is not necessarily a Vandermonde rank decomposition, even if $\operatorname{rank}_{V}(\mathcal{H})=\operatorname{rank}_{S}(\mathcal{H})$.

For each example, we not only find the Vandermonde decomposition of the given Hankel tensor $\mathcal{H}$, but also exhibit a symmetric decomposition of $\mathcal{H}$.

Example 3.6. Consider the Hankel matrix $\mathcal{H} \in \mathrm{H}^{2}\left(\mathbb{C}^{3}\right)$ :

$$
\mathcal{H}=\left(\mathcal{H}_{i j}\right)_{i, j=1}^{3}=\left[\begin{array}{lll}
0 & 0 & 1 \\
0 & 1 & 0 \\
1 & 0 & 0
\end{array}\right]
$$

We have $d=4, s=2$ and $h=(0,0,1,0,0)$. By Algorithm 3.4 we get $\operatorname{rank}_{V}(\mathcal{H})=\operatorname{rank}_{S}(h)=3$ and

$$
h(x, y)=6 x^{2} y^{2}=\sum_{i=1}^{3} \lambda_{i}\left(\alpha_{i} x+\beta_{i} y\right)^{4},
$$

where $($ denote $i:=\sqrt{-1})$

$$
\begin{aligned}
& \left(\lambda_{1}, \lambda_{2}, \lambda_{3}\right)=\left(\frac{1}{3},-\frac{1}{6}-\frac{\sqrt{3}}{6} i,-\frac{1}{6}+\frac{\sqrt{3}}{6} i\right), \\
& \left(\alpha_{1}, \alpha_{2}, \alpha_{3}\right)=\left(1, \frac{1+\sqrt{3} i}{2}, \frac{1-\sqrt{3} i}{2}\right) \\
& \left(\beta_{1}, \beta_{2}, \beta_{3}\right)=(1,-1,-1) .
\end{aligned}
$$

So, $\mathcal{H}$ has the Vandermonde rank decomposition

$$
\mathcal{H}=\sum_{i=1}^{3} \lambda_{i}\left(\beta_{i}^{2}, \alpha_{i} \beta_{i}, \alpha_{i}^{2}\right)^{\otimes 2} .
$$

On the other hand, $\mathcal{H}$ also has the symmetric rank decomposition

$$
\mathcal{H}=-\frac{1}{2}\left(e_{1}-e_{3}\right)^{\otimes 2}+\frac{1}{2}\left(e_{1}+e_{3}\right)^{\otimes 2}+e_{2}^{\otimes 2},
$$

where $\left\{e_{1}, e_{2}, e_{3}\right\}$ is the standard unit basis of $\mathbb{C}^{3}$. Indeed, $\operatorname{rank}_{S}(\mathcal{H})=3$, because $\mathcal{H}$ is a matrix and all the tensor ranks are the same.

Example 3.7. Let $\mathcal{H} \in \mathrm{H}^{3}\left(\mathbb{C}^{3}\right)$ be the Hankel tensor such that

$$
\mathcal{H}_{i j k}= \begin{cases}1, & \text { if } i+j+k=7, \\ 0, & \text { otherwise. }\end{cases}
$$

The vector $h=(0,0,0,0,1,0)$. In Algorithm 3.4 $s=3$ and rank $C_{3,3}(h)=3$. The unique (up to scaling) vector from the null space of $C_{3,3}(h)$ is $f=(1,0,0,0)$. The equation $f(x, y)=x^{3}=0$ has a triple root. Therefore, we obtain $\operatorname{rank}_{V}(\mathcal{H})=d-3+2=5$. Moreover, the matrix $C_{1,5}$ is

$$
C_{1,5}=\left[\begin{array}{llllll}
0 & 0 & 0 & 0 & 1 & 0 \\
0 & 0 & 0 & 1 & 0 & 0
\end{array}\right]
$$

and $g=\left[\begin{array}{llllll}1 & 0 & 0 & 0 & 0 & -1\end{array}\right]^{\top} \in \operatorname{Ker}\left(C_{1,5}\right)$. The binary form defined by $g$ is

$$
g(x, y)=x^{5}-y^{5}=\prod_{j=0}^{4}\left(x-w^{j} y\right), \quad w=\exp \left(\frac{2 \pi i}{5}\right) .
$$


We may obtain the linear system

$$
\left[\begin{array}{ccccc}
1 & w^{5} & w^{10} & w^{15} & w^{20} \\
5 & 5 w^{4} & 5 w^{8} & 5 w^{12} & 5 w^{16} \\
10 & 10 w^{3} & 10 w^{6} & 10 w^{9} & 10 w^{12} \\
10 & 10 w^{2} & 10 w^{4} & 10 w^{6} & 10 w^{8} \\
5 & 5 w & 5 w^{2} & 5 w^{3} & 5 w^{4} \\
1 & 1 & 1 & 1 & 1
\end{array}\right]\left[\begin{array}{l}
\lambda_{0} \\
\lambda_{1} \\
\lambda_{2} \\
\lambda_{3} \\
\lambda_{4}
\end{array}\right]=\left[\begin{array}{l}
0 \\
1 \\
0 \\
0 \\
0 \\
0
\end{array}\right]
$$

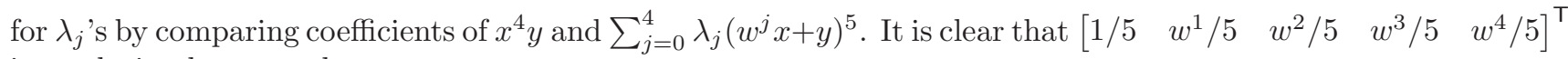
is a solution hence we have

$$
h(x, y)=5 x^{4} y=\sum_{j=0}^{4} \frac{w^{j}}{5}\left(w^{j} x+y\right)^{5}
$$

and

$$
\mathcal{H}=\sum_{j=0}^{4} w^{j}\left(1, w^{j}, w^{2 j}\right)^{\otimes 3} .
$$

On the other hand, the polynomial associated to $\mathcal{H}$ is $3\left(x z+y^{2}\right) z$, which has the Waring decomposition:

$$
\frac{1}{2}\left[-\frac{1}{2}(2 x-z)^{3}+\frac{8}{3}(x-z)^{3}+\frac{1}{6}(2 x+z)^{3}+(y+z)^{3}-(y-z)^{3}\right] .
$$

So, $\operatorname{rank}_{S}(\mathcal{H}) \leq 5$. In fact, $\operatorname{rank}_{S}(\mathcal{H})=5$ by [34, Table 1]. However, (3.4) is not a Vandermonde rank decomposition. In particular, this also implies that symmetric decompositions of $\mathcal{H}$ are not unique.

Example 3.8. Consider the Hankel tensor $\mathcal{H} \in \mathrm{H}^{m}\left(\mathbb{C}^{3}\right)$ be such that

$$
\mathcal{H}_{i_{1} \ldots i_{m}}= \begin{cases}1, & \text { if } i_{1}+\cdots+i_{m}=m+1 \text { or } 3 m \\ 0, & \text { otherwise. }\end{cases}
$$

The polynomial associated to $\mathcal{H}$ is $m x^{m-1} y+z^{m}, d=2 m$ and

$$
h_{l}= \begin{cases}1, & \text { if } l=1 \text { or } 2 m \\ 0, & \text { otherwise. }\end{cases}
$$

One can check that rank $C_{d-s, s}(h)=3$ and for $f \in \operatorname{ker} C_{2 m-3,3}(h)$ the polynomial $f(x, y)$ has a multiple root. Hence we have $\operatorname{rank}_{V}(\mathcal{H})=2 m-1$. On the other hand, by [34, Theorem 10.2], we know $m \leq$ $\operatorname{rank}_{S}(\mathcal{H}) \leq m+1$. Therefore, if $m \geq 3$, we have $\operatorname{rank}_{S}(\mathcal{H})<\operatorname{rank}_{V}(\mathcal{H})$.

\section{RANK RELATIONS FOR GENERAL ORDERS}

A Hankel tensor has the usual rank (i.e., the cp rank), symmetric rank, Vandermonde rank, border rank and symmetric border rank. This section studies relations among these various ranks for Hankel tensors. We discus them for the even and odd order cases separately. To do that, we first need to consider catalecticant matrices for Hankel tensors.

4.1. Catalecticant matrices. For the order $m$, let $m_{1}:=\lceil m / 2\rceil$. If $m=2 m_{0}$ is even, $m_{1}=m_{0}$; if $m=2 m_{0}+1$ is odd, $m_{1}=m_{0}+1$. A symmetric tensor can be flattened into catalecticant matrices [28]. Here, we consider the most square ones. For each $\mathcal{H} \in \mathrm{H}^{m}\left(\mathbb{C}^{n}\right)$, denote by Flat $(\mathcal{H})$ the matrix, whose row is indexed by an integral tuple $I=\left(i_{1} \ldots i_{m_{1}}\right)$ and whose column is indexed by another one $J=\left(i_{m_{1}+1}, \ldots, i_{m}\right)$, such that the entries of $\operatorname{Flat}(\mathcal{H})$ are given as

$$
\operatorname{Flat}(\mathcal{H})_{I, J}=\mathcal{H}_{i_{1} \ldots i_{m_{1}} i_{m_{1}+1} \ldots i_{m}} .
$$

Note that Flat $(\mathcal{H})$ is a $n^{m_{1}}-$ by $-n^{m-m_{1}}$ matrix. There is a correspondence between catalecticant matrices and symmetric flattenings for tensors [35, p.76]. Because $\mathcal{H}$ is Hankel, $\operatorname{Flat}(\mathcal{H})$ has repeated rows and columns. We consider a submatrix of $\operatorname{Flat}(\mathcal{H})$ that does not have repeated ones. Let $\mathrm{F}(\mathcal{H})$ be the $\operatorname{submatrix}$ of $\mathrm{Flat}(\mathcal{H})$ whose row index $I=\left(i_{1}, \ldots, i_{m_{1}}\right)$ is such that

$$
i_{1} \leq \cdots \leq i_{m_{1}}, \quad m_{1} \leq i_{1}+\cdots+i_{m_{1}} \leq n m_{1},
$$


and whose column index $J=\left(i_{m_{1}+1}, \ldots, i_{m}\right)$ is such that

$$
i_{m_{1}+1} \leq \cdots \leq i_{m}, \quad m-m_{1} \leq i_{m_{1}+1}+\cdots+i_{m} \leq n\left(m-m_{1}\right) .
$$

In the following, we give an expression for $\mathrm{F}(\mathcal{H})$.

Lemma 4.1. Let Flat $(\mathcal{H}), F(\mathcal{H})$ be as above. Let $l:=(n-1) m_{0}$. Then,

$$
F(\mathcal{H})=C_{d-l, l}(h),
$$

where $C_{d-l, l}(h)$ is defined as in (2.2). Moreover, rank $F(\mathcal{H})=\operatorname{rank} F l a t(\mathcal{H})$.

Proof. If $\mathcal{H}=\left(a^{n-1}, a^{n-2} b, \ldots, b^{n-1}\right)^{\otimes m}$ is rank-1, then

$$
\mathrm{F}(\mathcal{H})=\left[\begin{array}{ccccc}
a^{d} & a^{d-1} b & a^{d-2} b^{2} & \cdots & a^{d-l} b^{l} \\
a^{d-1} b & a^{d-2} b_{j}^{2} & a^{d-3} b^{3} & \cdots & a^{d-l-1} b^{l+1} \\
\vdots & \vdots & \vdots & \ddots & \vdots \\
a^{d-l} b^{l} & a^{d-l-1} b^{l+1} & a^{d-l-2} b^{l+2} & \cdots & b^{d}
\end{array}\right] .
$$

The equality (4.1) is clearly true. If $\mathcal{H}$ is not rank-1, then $\mathcal{H}$ is a sum of rank-1 Hankel tensors, say, $\mathcal{H}=$ $\mathcal{H}_{1}+\cdots+\mathcal{H}_{r}$, where each $\mathcal{H}_{i}$ is rank-1. As a matrix-valued function, $\mathrm{F}(\mathcal{H})$ is linear in $\mathcal{H}$, so

$$
\mathrm{F}(\mathcal{H})=\mathrm{F}\left(\mathcal{H}_{1}\right)+\cdots+\mathrm{F}\left(\mathcal{H}_{r}\right)=C_{d-l, l}(h) .
$$

Hence, (4.1) is true. Note that $\mathrm{F}(\mathcal{H})$ is the maximum submatrix of $\operatorname{Flat}(\mathcal{H})$ that does not have any repeated rows and columns. So, $\mathrm{F}(\mathcal{H})$ and $\operatorname{Flat}(\mathcal{H})$ have the same rank.

4.2. The even order case. When the order $m=2 m_{0}$ is even, the number $d:=(n-1) m$ is also even. So $s=\lfloor d / 2\rfloor=(n-1) m_{0}$. Recall that $h$ is determined by $\mathcal{H}$ as in (1.3) and $C_{d-s, s}(h)$ is the Hankel matrix determined by $h$ as in (2.2). By Theorem 2.2. the rank $r$ of $C_{d-s, s}$ is equal to the border rank of $h$, which also equals the Vandermonde border rank of $\mathcal{H}$. So, it always holds that

$$
\operatorname{brank}_{S}(h)=\operatorname{brank}_{V}(\mathcal{H})=r .
$$

If $r=s+1$, then $\operatorname{rank}_{V}(\mathcal{H})=r$ by Theorem 2.2 and if $r<s+1$, then there exists a unique (up to scaling) vector $0 \neq f \in \operatorname{ker} C_{d-r, r}(h)$. The rank relations are summarized as follows.

Theorem 4.2. Suppose $m=2 m_{0}$ is even. For $\mathcal{H} \in \mathrm{H}^{m}\left(\mathbb{C}^{n}\right)$, let $h, d, s, r, f$ be as above. Then, we have:

(i) If $r=s+1$, or if $r<s+1$ and $f$ has no multiple roots, then

$$
\operatorname{rank}_{V}(\mathcal{H})=\operatorname{rank}_{S}(\mathcal{H})=\operatorname{rank}(\mathcal{H})=\operatorname{brank}_{V}(\mathcal{H})=\operatorname{brank}_{S}(\mathcal{H})=\operatorname{brank}(\mathcal{H})=r
$$

(ii) If $r<s+1$ and $f$ has a multiple root, then

$$
d-r+2=\operatorname{rank}_{V}(\mathcal{H}) \geq \operatorname{rank}_{S}(\mathcal{H}) \geq \operatorname{brank}_{V}(\mathcal{H})=\operatorname{brank}_{S}(\mathcal{H})=\operatorname{brank}(\mathcal{H})=r .
$$

Proof. (i) If $r=d / 2+1$, or $r<d / 2+1$ and $f$ has no multiple roots, by Theorem 2.2 and Lemma 3.2

$$
r=\operatorname{rank}_{S}(h)=\operatorname{rank}_{V}(\mathcal{H}) \geq \operatorname{rank}_{S}(\mathcal{H}) .
$$

It is well-known that the border rank of a tensor is always greater than or equal to the rank of its flattening matrix [35]. Since $m=2 m_{0}$ is even, $s=l=(n-1) m_{0}$. By Lemma 4.1] we have

$$
\operatorname{brank}(\mathcal{H}) \geq \operatorname{rank} \operatorname{Flat}(\mathcal{H})=\operatorname{rank} \mathrm{F}(\mathcal{H})=\operatorname{rank} C_{d-s, s}(h)=r .
$$

Moreover, we also have

$$
\begin{gathered}
\operatorname{rank}_{S}(\mathcal{H}) \geq \operatorname{rank}(\mathcal{H}) \geq \operatorname{brank}(\mathcal{H}) \geq r \\
\operatorname{rank}_{S}(\mathcal{H}) \geq \operatorname{brank}_{S}(\mathcal{H}) \geq \operatorname{brank}(\mathcal{H}) \geq r .
\end{gathered}
$$

Since $r \geq \operatorname{rank}_{S}(\mathcal{H})$, all the ranks must be the same and the equalities in (4.3) hold.

(ii) If $r<d / 2+1$ and $f$ has a multiple root, then, by Theorem 2.2 and Lemma 3.2 , we have

$$
\operatorname{rank}_{V}(\mathcal{H})=\operatorname{rank}_{S}(h)=d-r+2>r .
$$

Note that the symmetric border rank of $h$ is $r$, by Theorem 2.2. Then, Lemma 3.2 implies that

$$
r=\operatorname{brank}_{S}(h) \geq \operatorname{brank}_{S}(\mathcal{H}) \geq \operatorname{brank}(\mathcal{H}) .
$$


As in the proof of (i), we can also prove that

$$
\operatorname{brank}_{S}(\mathcal{H}) \geq \operatorname{brank}(\mathcal{H}) \geq \operatorname{rank} \operatorname{Flat}(\mathcal{H}) \geq \operatorname{rank} \mathrm{F}(\mathcal{H})=r .
$$

Hence, (4.4) is true, because $\operatorname{rank}_{S}(\mathcal{H}) \geq \operatorname{brank}_{S}(\mathcal{H})$.

Theorem 4.2 immediately implies the following.

Corollary 4.3. If $\mathcal{H} \in \mathrm{H}^{m}\left(\mathbb{C}^{n}\right)$ is generic and $m$ is even, then its cp rank, symmetric rank, border rank, symmetric border rank, and Vandermonde rank are the same, which is $1+(n-1) m / 2$.

Proof. When $\mathcal{H}$ is generic, the vector $h$ is also generic and $\operatorname{rank} C_{s, s}(h)=d / 2+1$. By Theorem 4.2 (i), all the ranks are equal.

In particular, Theorem 4.2 also implies the following:

Corollary 4.4. For a generic Hankel tensor of an even order, Algorithm 3.4 produces a Vandermonde decomposition that achieves its cp rank, symmetric rank, border rank, symmetric boarder rank and Vandermonde rank simultaneously.

In the following, we give some examples to show applications of Theorem 4.2 In particular, we would like to remark that:

1) It is possible that $\operatorname{rank}_{V}(\mathcal{H})>\operatorname{rank}_{S}(\mathcal{H})$, even if the order is even.

2) We may have $\operatorname{rank}_{V}(\mathcal{H})=\operatorname{rank}_{S}(\mathcal{H})>\operatorname{brank}_{S}(\mathcal{H})$.

Example 4.5. Consider the Hankel tensor $\mathcal{H}$ in Example 3.8, We have

$$
\operatorname{rank}_{V}(\mathcal{H})=2 m-1, \quad m \leq \operatorname{rank}_{S}(\mathcal{H}) \leq m+1, \quad \operatorname{brank}_{S}(\mathcal{H})=3 .
$$

If $m \geq 4$, then $\operatorname{brank}_{S}(\mathcal{H})<\operatorname{rank}_{S}(\mathcal{H})<\operatorname{rank}_{V}(\mathcal{H})$.

Example 4.6. Consider the Hankel tensor $\mathcal{H} \in \mathrm{H}^{m}\left(\mathbb{C}^{3}\right)$ such that

$$
\mathcal{H}_{i_{1}, \ldots, i_{m}}= \begin{cases}1, & \text { if } i_{1}+\cdots+i_{m}=m+2 \\ 0, & \text { otherwise. }\end{cases}
$$

The polynomial associated to $\mathcal{H}$ is $\left(\begin{array}{c}m \\ 2\end{array}\right) x^{m-2} y^{2}+m x^{m-1} z$. By Algorithm 3.4 and Theorem 4.2, $\operatorname{rank}_{V}(\mathcal{H})=$ $2 m-1$. By [34, Theorem 10.2],

$$
\operatorname{brank}_{S}(\mathcal{H})=3, \quad m \leq \operatorname{rank}_{S}(\mathcal{H}) \leq 2 m-1 .
$$

If $m \geq 4, \operatorname{brank}_{S}(\mathcal{H})<\operatorname{rank}_{S}(\mathcal{H})$.

4.3. The odd order case. When the order $m=2 m_{0}+1$ is odd, the number $d=(n-1) m$ might not be even, and $s=\lfloor d / 2\rfloor$ might be different from $(n-1) m_{0}$. For $\mathcal{H} \in \mathrm{H}^{m}\left(\mathbb{C}^{n}\right), h$ is still the vector as in (1.3) and $r=\operatorname{rank} C_{d-s, s}(h)$. Like (4.2), it also holds that

$$
\operatorname{brank}_{S}(h)=\operatorname{brank}_{V}(\mathcal{H})=r .
$$

Let $f=\left(f_{0}, \ldots, f_{r}\right) \in \mathbb{C}^{r+1}$ be the unique vector (up to scaling) in $\operatorname{ker} C_{d-r, r}(h)$. The rank relations for $\mathcal{H}$ are as follows.

Theorem 4.7. Let $n, m, d, \mathcal{H}, h, r, f$ be as above. Suppose $r \leq 1+(n-1) m_{0}$, then we have:

(i) If $f(x, y)$ has no multiple roots, then

(ii) If $f(x, y)$ has a multiple root, then

$$
d-r+2=\operatorname{rank}_{V}(\mathcal{H}) \geq \operatorname{rank}_{S}(\mathcal{H}) \geq \operatorname{brank}_{V}(\mathcal{H})=\operatorname{brank}_{S}(\mathcal{H})=\operatorname{brank}(\mathcal{H})=r .
$$

Proof. We follow the same approach as in the proof of Theorem 4.2. The difference is that we need the assumption that $r \leq 1+(n-1) m_{0}$ when the order $m$ is odd.

(i) When $f$ has no multiple roots, by Theorem 2.2 and Lemma 3.2, we also have

$$
r=\operatorname{rank}_{S}(h)=\operatorname{rank}_{V}(\mathcal{H}) \geq \operatorname{rank}_{S}(\mathcal{H}) .
$$


Let $l:=(n-1) m_{0}$. In the following, we show that

$$
\text { rank } C_{d-l, l}(h)=r \text {. }
$$

Note that $r \leq 1+l$, rank $C_{d-s, s}(h)=r$, and $\operatorname{brank}_{S}(h)=r$ by Theorem 2.2

- When $r \leq l$, (4.7) is true by Proposition 9.7 of 24, since $\operatorname{brank}_{S}(h)=r$.

- When $r=1+l$, we still have rank $C_{d-l, l}(h) \leq r$. If rank $C_{d-l, l}(h)<r$, then $\operatorname{brank}_{S}(h)<r$ by Proposition 9.7 of 24, which is a contradiction. So, 4.7] is also true.

Recall the matrices Flat $(\mathcal{H}), \mathrm{F}(\mathcal{H})$ defined as in the subsection 4.1. By Lemma 4.1,

$$
\operatorname{brank}(\mathcal{H}) \geq \operatorname{rank} \operatorname{Flat}(\mathcal{H})=\operatorname{rank} \mathrm{F}(\mathcal{H})=\operatorname{rank} C_{d-l, l}(h)=r .
$$

Also note that $\operatorname{brank}(\mathcal{H}) \leq \operatorname{brank}_{S}(\mathcal{H}) \leq \operatorname{rank}_{S}(\mathcal{H})$. Since $\operatorname{rank}_{V}(\mathcal{H})=r$, the relation (1.5) and the above imply that all the ranks must be the same.

(ii) The proof is the same as for item (ii) of Theorem 4.2

When $\mathcal{H}$ is generic and $m=2 m_{0}+1$, for $n>2$, we have

$$
r=(n-1) m_{0}+\lceil n / 2\rceil>1+(n-1) m_{0} .
$$

Hence, the rank relations in Theorem 4.7 are not guaranteed any more. However, we can still get a lower and upper bound for those ranks.

Proposition 4.8. If the order $m=2 m_{0}+1$ is odd and $\mathcal{H} \in \mathrm{H}^{m}\left(\mathbb{C}^{n}\right)$ is generic, then

$$
\begin{gathered}
\operatorname{rank}_{V}(\mathcal{H})=m_{0}(n-1)+\lceil n / 2\rceil \text {, and } \\
m_{0}(n-1)+1 \leq \operatorname{brank}(\mathcal{H}) \leq \operatorname{rank}(\mathcal{H}) \leq \operatorname{rank}_{S}(\mathcal{H}) \leq \operatorname{rank}_{V}(\mathcal{H}) .
\end{gathered}
$$

Proof. By Proposition 3.3. we know that $\operatorname{rank}_{V}(\mathcal{H})=m_{0}(n-1)+\lceil n / 2\rceil$. The latter three inequalities are obvious. It is enough to prove the first one. We follow the proof of item (i) in Theorem 4.7 For all $\mathcal{H}$, we always have $\left(l=(n-1) m_{0}\right)$ :

$$
\operatorname{brank}(\mathcal{H}) \geq \operatorname{rank} \operatorname{Flat}(\mathcal{H}) \geq \operatorname{rank} \mathrm{F}(\mathcal{H})=C_{d-l, l}(h) .
$$

When $\mathcal{H}$ is generic, $h$ is also generic and so rank $C_{d-l, l}(h)=1+l$, which completes the proof.

\section{RANK RELATIONS FOR ORDER THREE}

This section studies the relations among various ranks of Hankel tensors when the order $m=3$. For cubic Hankel tensors, we are able to get better rank relations, in addition to those given in Theorem 4.7 Recall that for each tensor $T$ and positive integer $p$, a linear map $\varphi_{T}^{p}$ is defined in the subsection 2.2 for vector spaces $A=B=C=\mathbb{C}^{n}$. We use the standard unit vector basis $\left\{e_{1}, \ldots, e_{n}\right\}$ for $\mathbb{C}^{n}$ and we identify $\mathbb{C}^{n}$ with its dual so that $\left\{e_{1}, \ldots, e_{n}\right\}$ is also a dual basis for itself. A tensor $T \in \mathbb{C}^{n} \otimes \mathbb{C}^{n} \otimes \mathbb{C}^{n}$ can be written as

$$
T=\sum_{i, j, k=1}^{n} t_{i j k} e_{i} \otimes e_{j} \otimes e_{k}, \quad t_{i j k} \in \mathbb{C} .
$$

Let $1 \leq p \leq n-1$ be an integer. From (2.1) the linear map

$$
\varphi_{T}^{p}:\left(\bigwedge^{p} \mathbb{C}^{n}\right) \otimes\left(\mathbb{C}^{n}\right)^{*} \rightarrow\left(\bigwedge^{p+1} \mathbb{C}^{n}\right) \otimes \mathbb{C}^{n}
$$

is defined by setting

$$
\left(e_{k_{1}} \wedge \cdots \wedge e_{k_{p}}\right) \otimes e_{i} \mapsto \sum_{j, k=1}^{n} t_{i j k}\left(e_{k_{1}} \wedge \cdots \wedge e_{k_{p}} \wedge e_{j}\right) \otimes e_{k}
$$

and extending it linearly. By Theorem 2.1, we have

$$
\operatorname{brank}(T) \geq \operatorname{rank} \varphi_{T}^{p} /\left(\begin{array}{c}
n-1 \\
p
\end{array}\right) .
$$

We construct the representing matrix $M:=M_{T}^{p}$ for the linear map $\varphi_{T}^{p}$ under the standard basis. The set

$$
\left\{\left(e_{k_{1}} \wedge \cdots \wedge e_{k_{p}}\right) \otimes e_{i}: 1 \leq k_{1}<\cdots<k_{p} \leq n, 1 \leq i \leq n\right\}
$$


is a basis of $\left(\bigwedge^{p} \mathbb{C}^{n}\right) \otimes\left(\mathbb{C}^{n}\right)^{*}$ and

$$
\left\{\left(e_{k_{1}} \wedge \cdots \wedge e_{k_{p+1}}\right) \otimes e_{k}: 1 \leq k_{1}<\cdots<k_{p+1} \leq n, 1 \leq k \leq n\right\}
$$

is a basis of $\left(\bigwedge^{p+1} \mathbb{C}^{n}\right) \otimes \mathbb{C}^{n}$. The representing matrix $M:=M_{T}^{p}$ for $\varphi_{T}^{p}$ is $n\left(\begin{array}{c}n \\ p\end{array}\right) \times n\left(\begin{array}{c}n \\ p+1\end{array}\right)$ 可 We label the rows of $M$ by

and label the columns of $M$ by

$$
(J, i):=\left(j_{1}<\cdots<j_{p}, i\right)
$$

$$
\left(J^{\prime}, k\right):=\left(j_{1}^{\prime}<\cdots<j_{p+1}^{\prime}, k\right) .
$$

The entry of $M$ on the $(J, i)$-th row and $\left(J^{\prime}, k\right)$-th column is

$$
M_{(J, i),\left(J^{\prime}, k\right)}=\epsilon_{J, J^{\prime}, j} t_{i j k},
$$

where

$$
\epsilon_{J, J^{\prime}, j}= \begin{cases}(-1)^{p-q}, & \text { if } j_{1}^{\prime}=j_{1}, \ldots, j_{q-1}^{\prime}=j_{q-1}, \text { and } \\ & j_{q}^{\prime}=j, j_{q+1}^{\prime}=j_{q}, \ldots, j_{p+1}^{\prime}=j_{p} \\ 0, & \text { otherwise. }\end{cases}
$$

The following is an example of $M_{T}^{p}$ when $T$ is a cubic Hankel tensor.

Example 5.1. Consider the linear map $\varphi_{\mathcal{H}}^{1}: \mathbb{C}^{3} \otimes\left(\mathbb{C}^{3}\right)^{*} \rightarrow\left(\wedge^{2} \mathbb{C}^{3}\right) \otimes \mathbb{C}^{3}$ for a Hankel tensor $\mathcal{H} \in \mathrm{H}^{3}\left(\mathbb{C}^{3}\right)$. Note that

$$
\varphi_{\mathcal{H}}^{1}\left(e_{j} \otimes e_{i}\right)=\sum_{j^{\prime}, k=1}^{3} \mathcal{H}_{i j^{\prime} k}\left(e_{j} \wedge e_{j^{\prime}}\right) \otimes e_{k} .
$$

Let $h$ be the vector as in (1.3), then

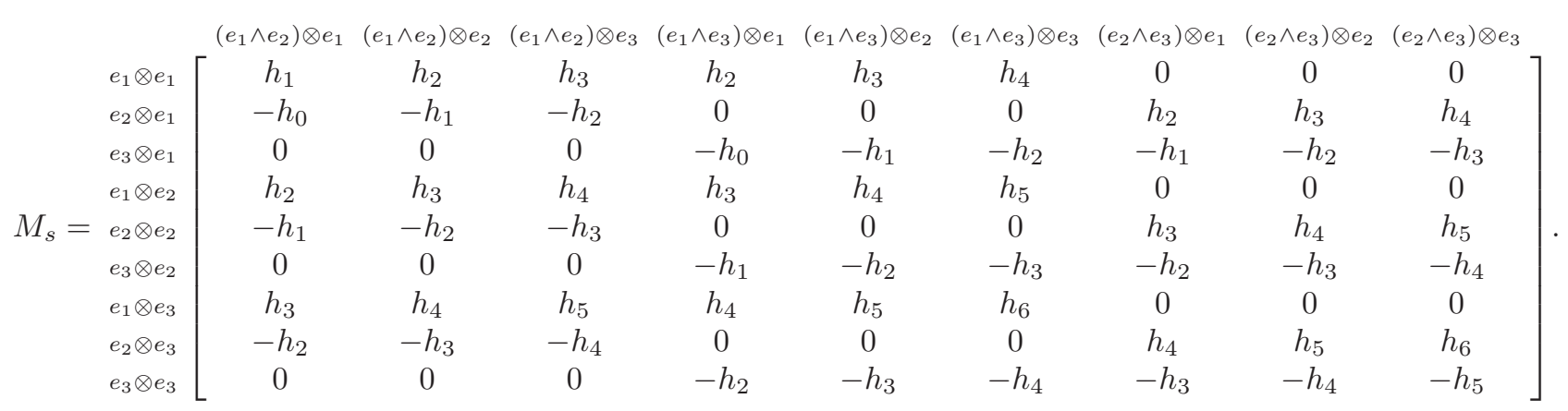

One can verify that rank $M_{\mathcal{H}}^{1}=8$ when $\mathcal{H}$ is generic. Indeed, the sum of the third and the seventh column is equal to the fifth column of $M_{\mathcal{H}}^{1}$, which implies that rank $M_{\mathcal{H}}^{1} \leq 8$. Moreover, it is easy to verify that the submatrix obtained by removing the seventh column from $M_{\mathcal{H}}^{1}$ has full rank 8 . Indeed, after a permutation on rows, the matrix $M_{\mathcal{H}}^{1}$ corresponds to the matrix in (3.8.1) of [35, page 81] and the Koszul flattening matrix in [48, Example 4.2]. By Theorem[2.1, we can get brank $(\mathcal{H}) \geq 4$. If $\varphi_{\mathcal{H}}^{2}$ is used, we get another lower bound for $\operatorname{brank}(\mathcal{H})$. However, rank $M_{\mathcal{H}}^{2} \leq 3$, which is worse than the one by using $\varphi_{\mathcal{H}}^{1}$.

Theorem 5.2. For a generic Hankel tensor $H \in S^{3}\left(\mathbb{C}^{n}\right)$, its border rank is at least $\left\lfloor\frac{3 n-1}{2}\right\rfloor$.

Proof. Let $r:=\left\lfloor\frac{3 n-1}{2}\right\rfloor$ and $p:=\left\lfloor\frac{n}{2}\right\rfloor$. By Theorem 2.1 it is sufficient to prove that the rank of the linear $\operatorname{map} \varphi_{\mathcal{H}}^{p}:\left(\bigwedge^{p} \mathbb{C}^{n}\right) \otimes\left(\mathbb{C}^{n}\right)^{*} \rightarrow\left(\bigwedge^{p+1} \mathbb{C}^{n}\right) \otimes \mathbb{C}^{n}$ has rank $\left(\begin{array}{c}n-1 \\ p\end{array}\right) r$. By the lower semi-continuity ${ }^{6}$ of the matrix rank function, it is sufficient to prove that $\operatorname{rank}\left(M_{\mathcal{H}}^{p}\right) \geq\left(\begin{array}{c}n-1 \\ p\end{array}\right) r$ for some order three Hankel tensor $\mathcal{H}$. To do this, we take $\mathcal{H}=\left(\mathcal{H}_{i j k}\right)$, where

$$
\mathcal{H}_{i j k}= \begin{cases}1, & \text { if } i+j+k-2=r, \\ 0, & \text { otherwise }\end{cases}
$$

\footnotetext{
5 One can also take the transpose to obtain an $n\left(\begin{array}{c}n \\ p+1\end{array}\right) \times n\left(\begin{array}{c}n \\ p\end{array}\right)$ matrix. Since we only concern the rank, both matrices are okay for the proof.

6 To be more precise, if $M\left(x_{1}, \ldots, x_{k}\right)$ is an $m \times n$ matrix polynomial and rank $M\left(a_{1}, \ldots, a_{k}\right)=r$ for some $\left(a_{1}, \ldots, a_{k}\right) \in \mathbb{C}^{k}$, then there exists a Zariki open dense subset $U$ of $\mathbb{C}^{k}$ such that $\operatorname{rank} M\left(b_{1}, \ldots, b_{k}\right) \geq r$ for all $\left(b_{1}, \ldots, b_{k}\right) \in U$.
} 
The tensor $\left(e_{j_{1}} \wedge \cdots \wedge e_{j_{p}}\right) \otimes e_{i}$ is mapped by $\varphi_{\mathcal{H}}^{p}$ to

$$
\sum_{j=1}^{n}(-1)^{p-q}\left(e_{j_{1}} \wedge \cdots \wedge e_{j_{q}} \wedge e_{j} \wedge e_{j_{q+1}} \wedge \cdots \wedge e_{j_{p}}\right) \otimes e_{r+2-i-j}
$$

where the summation is over

$$
1 \leq j_{1}<\cdots<j_{q}<j<j_{q+1}<\cdots<j_{p} \leq n .
$$

We set $e_{r+2-i-j}=0$ if $i+j \geq r+2$ or $i+j \leq\left\lfloor\frac{n+1}{2}\right\rfloor$. Hence, the summand in (5.2) is non-zero if and only if

$$
\max \{1, r-i-n+2\} \leq j \leq \min \{n, r-i+1\} \quad \text { and } j \notin\left\{j_{1}, \ldots, j_{p}\right\} .
$$

For given $j_{1}<\cdots<j_{q}<j<j_{q+1}<\cdots<j_{p}$, we let

$$
J:=\left(j_{1}<\cdots<j_{p}\right) \text { and } J^{\prime}:=\left(j_{1}<\cdots<j_{q}<j<j_{q+1}<\cdots<j_{p}\right) .
$$

By (5.2), the matrix $M_{\mathcal{H}}^{p}$ has a block that is

$$
\begin{gathered}
\multicolumn{10}{c}{T_{J, J^{\prime}}:=\epsilon_{J, J^{\prime}} T_{j-\left\lfloor\frac{n+1}{2}\right\rfloor}=} \\
(J, 1) \\
(J, 2) \\
\vdots \\
(J, r-j+1) \\
\vdots \\
(J, n-1) \\
(J, n)
\end{gathered}\left[\begin{array}{cccccccc}
\left(J^{\prime}, n\right) & \left(J^{\prime}, n-1\right) & \cdots & \left(J^{\prime}, r-j+2\right) & \left(J^{\prime}, r-j+1\right) & \left(J^{\prime}, r-j\right) & \cdots & \left(J^{\prime}, 1\right) \\
0 & 0 & \cdots & 0 & (-1)^{p-q} & 0 & \cdots & 0 \\
0 & 0 & \cdots & 0 & 0 & (-1)^{p-q} & \cdots & 0 \\
0 & \vdots & \ddots & \vdots & \vdots & \vdots & \ddots & \vdots \\
0 & 0 & \cdots & 0 & 0 & 0 & \cdots & (-1)^{p-q} \\
0 & 0 & \cdots & 0 & 0 & 0 & \cdots & 0 \\
& 0 & \cdots & 0 & 0 & 0 & \cdots & 0
\end{array}\right],
$$

where $\epsilon_{J, J^{\prime}}=(-1)^{p-q}$ and $T_{j-\left\lfloor\frac{n+1}{2}\right\rfloor}$ is the $n \times n$ Toeplitz matrix whose $(i, k)$-th entry $t_{i k}$ is defined by

$$
t_{i k}= \begin{cases}1, & \text { if } k-i=j-\left\lfloor\frac{n+1}{2}\right\rfloor \\ 0, & \text { otherwise. }\end{cases}
$$

Clearly, we have

$$
\operatorname{rank}\left(T_{J, J^{\prime}}\right)= \begin{cases}r-j+1, & \text { if } r-j+1 \leq n, \\ 2 n-(r-j+1), & \text { otherwise. }\end{cases}
$$

In particular, if $J^{\prime}=J \cup\left\{\left\lfloor\frac{n+1}{2}\right\rfloor\right\}$, then $\operatorname{rank}\left(T_{J, J^{\prime}}\right)=n$, i.e., the matrix $T_{J, J^{\prime}}$ is the identity matrix up to a sign. Let $C_{0}$ be the set of sequences $J^{\prime}=\left(j_{1}<\cdots<j_{p+1}\right)$ such that $\left\lfloor\frac{n+1}{2}\right\rfloor \in J^{\prime}$ and let $R_{0}$ be the set of sequences $J=\left(j_{1}<\cdots<j_{p}\right)$ such that $\left\lfloor\frac{n+1}{2}\right\rfloor \notin J$. For each $J \in R_{0}$, there is a unique $J^{\prime} \in C_{0}$ such that $J^{\prime}=J \cup\left\{\left\lfloor\frac{n+1}{2}\right\rfloor\right\}$. We also let $C$ be the set of sequences $J^{\prime}=\left(j_{1}<\cdots<j_{p+1}\right)$ such that $\left\lfloor\frac{n+1}{2}\right\rfloor \notin J^{\prime}$ and let $R$ be the set of sequences $J=\left(j_{1}<\cdots<j_{p}\right)$ such that $\left\lfloor\frac{n+1}{2}\right\rfloor \in J$. Then the matrix $M_{\mathcal{H}}^{p}$ may be visualized as follows:

$$
\left.M_{\mathcal{H}}^{p}={ }_{R}^{R_{0}} \begin{array}{cc}
C_{0} & C \\
D & T_{1} \\
T_{2} & 0
\end{array}\right],
$$

where $D$ is the submatrix of $M_{\mathcal{H}}^{p}$ obtained by taking $J$ 's from $R_{0}$ and $J^{\prime}$ 's from $C_{0}$ and $T_{1}, T_{2}$ are defined in the same way. Since for each $J \in R_{0}$ there exists the unique $J^{\prime} \in C_{0}$ such that $J^{\prime}=J \cup\left\{\left\lfloor\frac{n+1}{2}\right\rfloor\right\}$, we see that $D$ is actually a block diagonal matrix where each diagonal block is of the form $T_{J, J^{\prime}}$, which is the $n \times n$ identity matrix (up to a sign) because $J^{\prime}=J \cup\left\{\left\lfloor\frac{n+1}{2}\right\rfloor\right\}$. Indeed, $D$ is a diagonal matrix whose diagonal entries are either 1 or -1 . Moreover, the cardinality of both $C_{0}$ and $R_{0}$ is equal to

$$
\# C_{0}=\# R_{0}=\left(\begin{array}{c}
n-1 \\
p
\end{array}\right)
$$

this implies that $D$ is a full rank $\left(\begin{array}{c}n-1 \\ p\end{array}\right) n \times\left(\begin{array}{c}n-1 \\ p\end{array}\right) n$ matrix. 
Next, we apply column operations to $M_{\mathcal{H}}^{p}$ to make it a triangular matrix. More precisely, we compute

$$
M_{\mathcal{H}}^{p}\left[\begin{array}{cc}
\operatorname{Id}_{\left(\begin{array}{c}
n-1 \\
p
\end{array}\right) n} & -D^{-1} T_{1} \\
0 & \operatorname{Id}_{\left(\begin{array}{c}
n-1 \\
p+1
\end{array}\right) n}
\end{array}\right]=\left[\begin{array}{cc}
D & 0 \\
T_{2} & -T_{2} D^{-1} T_{1}
\end{array}\right]=\left[\begin{array}{cc}
D & 0 \\
T_{2} & -T_{2} D T_{1}
\end{array}\right] .
$$

Here $\operatorname{Id}_{m}$ is the $m \times m$ identity matrix and the second equality follows from the fact that $D$ is a diagonal matrix whose diagonal entries are 1 or -1 . We denote by $M$ the matrix $-T_{2} D T_{1}$ and by Lemma 5.3 , we see that

$$
\operatorname{rank} M=\left(\begin{array}{c}
n-1 \\
p+1
\end{array}\right)(p+1) .
$$

Therefore, $M_{\mathcal{H}}^{p}$ has the rank

$$
\operatorname{rank} M_{\mathcal{H}}^{p}=\left(\begin{array}{c}
n-1 \\
p
\end{array}\right) n+\left(\begin{array}{c}
n-1 \\
p+1
\end{array}\right)(p+1)=\left(\begin{array}{c}
n-1 \\
p
\end{array}\right) r
$$

Lemma 5.3. Let $\mathcal{H}, M_{\mathcal{H}}^{p}, T_{1}, T_{2}, D$ and $M$ be as in the proof of Theorem 5.2. The rank of $M$ is equal to $\left(\begin{array}{c}n-1 \\ p+1\end{array}\right)(p+1)$.

The proof of Lemma 5.3 will be given in the Appendix.

Theorem 5.4. For a generic Hankel tensor $\mathcal{H} \in \mathrm{H}^{3}\left(\mathbb{C}^{n}\right)$, we have

$$
\operatorname{brank}(\mathcal{H})=\operatorname{brank}_{S}(\mathcal{H})=\operatorname{rank}(\mathcal{H})=\operatorname{rank}_{S}(\mathcal{H})=\operatorname{rank}_{V}(\mathcal{H})=\left\lfloor\frac{3 n-1}{2}\right\rfloor
$$

Proof. By Theorem 3.3, we have seen that $\operatorname{rank}_{V}(\mathcal{H})$ is

$$
\left\lceil\frac{3 n-2}{2}\right\rceil=\left\lceil\frac{3 n}{2}\right\rceil-1=\left\lfloor\frac{3 n-1}{2}\right\rfloor .
$$

Theorem 5.2 implies that $\operatorname{brank}(\mathcal{H}) \geq\left\lfloor\frac{3 n-1}{2}\right\rfloor$ when $\mathcal{H}$ is generic. Since we always have

$$
\begin{gathered}
\operatorname{brank}(\mathcal{H}) \leq \operatorname{rank}(\mathcal{H}) \leq \operatorname{rank}_{S}(\mathcal{H}) \leq \operatorname{rank}_{V}(\mathcal{H}), \\
\operatorname{brank}(\mathcal{H}) \leq \operatorname{brank}_{S}(\mathcal{H}) \leq \operatorname{rank}_{S}(\mathcal{H})
\end{gathered}
$$

the conclusion follows directly.

Theorem 5.4 clearly implies the following.

Corollary 5.5. For a generic Hankel tensor of order three, Algorithm 3.4 gives a decomposition that achieves its cp rank, symmetric rank, border rank, symmetric border rank, and Vandermonde rank.

In Theorem [5.4, we can get concrete conditions for the equalities there to hold. In Algorithm 3.4 by (1.5) and Theorem 2.1, we know (5.3) holds if

- $\operatorname{rank} M_{\mathcal{H}}^{p} \geq\lfloor(3 n-1) / 2\rfloor\left(\begin{array}{c}n-1 \\ p\end{array}\right)$;

- when $n$ is odd, $\operatorname{rank} C_{d-s, s}(h)=1+s$;

- when $n$ is even, rank $C_{d-s, s}(h)=1+s$ and the binary form $f(x, y)$ has no multiple roots.

In the following, we give some examples that the conclusion of Theorem 5.4 may not hold for non-generic Hankel tensors.

Example 5.6. Consider the Hankel tensor $\mathcal{H} \in \mathrm{H}^{3}\left(\mathbb{C}^{3}\right)$ such that

$$
\mathcal{H}_{i j k}= \begin{cases}1, & \text { if } i+j+k=8, \\ 0, & \text { otherwise. }\end{cases}
$$

The polynomial associated to $\mathcal{H}$ is $3 y z^{2}$. We have $d=6, s=3, h=(0,0,0,0,0,1,0)$ and $r=\operatorname{rank} C_{d-s, s}=2$. By Algorithm 3.4, we get $\operatorname{rank}_{V}(\mathcal{H})=6$. However, $\operatorname{rank}_{S}(\mathcal{H})=3$ and $\operatorname{brank}_{S}(\mathcal{H})=2$ by [34, Table 1] or [49, Theorem $1.1 \& 1.2]$. However,

$$
\frac{3 n-1}{2}=4>\operatorname{rank}\left(\varphi_{\mathcal{H}}^{1}\right)=2 .
$$


Hence, $\operatorname{brank}(\mathcal{H})=2$ and $\operatorname{rank}(\mathcal{H})=2$ or 3 . In fact, $\operatorname{rank}(\mathcal{H})=3$. If otherwise $\operatorname{rank}(\mathcal{H})=2$, then $\mathcal{H}$ has a decomposition

$$
\mathcal{H}=u_{1} \otimes v_{1} \otimes w_{1}+u_{1} \otimes v_{2} \otimes w_{2}, \quad u_{i}, v_{i}, w_{i} \in \mathbb{C}^{3}, i=1,2 .
$$

One may use Macaulay2 223 to verify that such a decomposition does not exist.

Example 5.7. Let $\mathcal{H}$ be the Hankel tensor as in Example 3.8 for $m=3$. We know $\operatorname{rank}_{V}(\mathcal{H})=5$. By 34 , Table 1],

Moreover, $\operatorname{rank}\left(\varphi_{\mathcal{H}}^{1}\right)=3$, hence

$$
\operatorname{rank}_{S}(\mathcal{H})=4, \quad \operatorname{brank}_{S}(\mathcal{H})=3
$$

$$
\operatorname{brank}(\mathcal{H})=3, \quad \operatorname{rank}(\mathcal{H})=3 \text { or } 4 .
$$

Since the monomials $x^{2} y$ and $z^{3}$ do not share a common variable, by [35, Sec. 9.1.4], we have

$$
\operatorname{rank}(\mathcal{H})=\operatorname{rank}\left(x^{2} y\right)+\operatorname{rank}\left(z^{3}\right)=3+1=4=\operatorname{rank}_{S}(\mathcal{H}) .
$$

Example 5.8. Consider the special case of Example 4.6 with $m=3$. We have seen that $\operatorname{rank}_{V}(\mathcal{H})=5$, $\operatorname{brank}_{S}(\mathcal{H})=3$. By [34, Theorem 10.2], $\operatorname{rank}_{S}(\mathcal{H})=5$. One can check that $\operatorname{rank}\left(\varphi_{\mathcal{H}}^{1}\right)=3$, hence

$$
3=\operatorname{brank}(\mathcal{H})=\operatorname{brank}_{S}(\mathcal{H})<\operatorname{rank}_{V}(\mathcal{H})=5 .
$$

This implies that $\operatorname{rank}(\mathcal{H})=3,4$ or 5 . Indeed, we may verify again by Macaulay2 23$]$ that $\operatorname{rank}(\mathcal{H})=5=$ $\operatorname{rank}_{S}(\mathcal{H})$.

\section{Conclusions and Questions}

The main results of this article are:

1) We give an algorithm (Algorithm 3.4) for computing Vandermonde rank decompositions for all Hankel tensors. In particular, the Vandermonde rank of a generic $\mathcal{H} \in \mathrm{H}^{m}\left(\mathbb{C}^{n}\right)$ is $\left\lceil\frac{m(n-1)+1}{2}\right\rceil$ (Proposition 3.3).

2) We can determine the cp rank, symmetric rank, border rank and symmetric border rank of a Hankel tensor, under some concrete conditions (Theorem 4.2. Theorem 4.7).

3) We prove that the cp rank, symmetric rank, border rank, symmetric border rank and Vandermonde rank are all the same for a generic Hankel tensor of order even or three (Corollary 4.3 , Theorem 5.4).

However, we do not know much about the rank relations for generic Hankel tensors of odd order $m \geq 5$. Naturally, we pose the following question:

Question 6.1. For an odd order $m \geq 5$ and for a generic Hankel tensor $\mathcal{H} \in \mathrm{H}^{m}\left(\mathbb{C}^{n}\right)$, do we have

$$
\operatorname{rank}(\mathcal{H})=\operatorname{rank}_{S}(\mathcal{H})=\operatorname{brank}(\mathcal{H})=\operatorname{brank}_{S}(\mathcal{H})=\operatorname{rank}_{V}(\mathcal{H}) ?
$$

We point out that the answer to Question 6.1 is "no" if we replace "generic" by "all", as we have already seen in the earlier examples. However, we conjecture that the answer to Question 6.1] is yes.

Conjecture 6.2. The answer to Question 6.1 is yes.

Finally, we conjecture that Comon's conjecture remains true at least for Hankel tensors, although Y. Shitov provides a counterexample in [56] which implies that Comon's conjecture does not hold for all symmetric tensors.

Conjecture 6.3. For all $\mathcal{H} \in \mathrm{H}^{m}\left(\mathbb{C}^{n}\right), \operatorname{rank}(\mathcal{H})=\operatorname{rank}_{S}(\mathcal{H})$.

Acknowledgement The authors would like to thank Lek-Heng Lim and two anonymous referees for their fruitful suggestions on improving the paper. Jiawang Nie was partially supported by the NSF grants DMS1417985 and DMS-1619973. Ke Ye was partially supported by National Key R\&D Program of China Grant no. 2018YFA0306702, the NSFC grant NSFC No.11688101, the Hundred Talents Program of the Chinese Academy of Sciences as well as the Recruitment Program of Global Experts of China.

7 34. Theorem 10.2] states that $m \leq \operatorname{rank}_{S}(\mathcal{H}) \leq 2 m-1$ in general, but by the remark after Theorem $10.2, \operatorname{rank}_{S}(\mathcal{H})$ attains the upper bound 5 if $m=3$. 


\section{REFERENCES}

[1] J. Alexander and A. Hirschowitz, "Polynomial interpolation in several variables," Journal of Algebraic Geometry 4.4, pp. 201-222, 1995.

[2] E. Ballico and A. Bernardi, "Tensor ranks on tangent developable of Segre varieties," Linear and Multilinear Algebra, issue. 1, pp. 1-14, 2012.

[3] A. Bernardi, J. Brachat, P. Comon and B. Mourrain, "General tensor decomposition, moment matrices and applications", J. Symbolic Comput. 52, pp. 51-71, 2013.

[4] A. Bernardi, A. Gimigliano and M. Idà, "Computing symmetric rank for symmetric tensors", Journal of Symbolic Computation 46, 34-53, 2011.

[5] J. Brachat, P. Comon, B. Mourrain and E. Tsigaridas, "Symmetric tensor decomposition", Linear Algebra Appl. 433, no. 11-12, pp. 1851-1872, 2010.

[6] P. Bürgisser, M. Clausen and M. A. Shokrollahi, Algebraic Complexity Theory, Grundlehren der Mathematischen Wissenschaften, 315, Springer-Verlag, Berlin, 1997.

[7] H. Chen, G. Li, and L. Qi, "Further results on Cauchy tensors and Hankel tensors", Appl. Math. Comput. 275, pp. 50-62, 2016.

[8] Y. Chen, L. Qi, and Q. Wang, "Computing extreme eigenvalues of large scale Hankel tensors", J. Sci. Comput. 68, no. 2, pp. 716-738, 2016.

[9] L. Chiantini, G. Ottaviani, and N. Vannieuwenhoven, "On generic identifiability of symmetric tensors of subgeneric rank", Transactions of the American Mathematical Society 369. 6, pp. 4021-4042, 2017.

[10] G. Comas and M. Seiguer, "On the rank of a binary form", Found. Comput. Math., vol. 11, pp. 65-78, 2011.

[11] P. Comon, Tensor decompositions in Mathematics in Signal Processing (J. G. McWhirter and I. K. Proudler, eds.), Clarendon Press, Oxford, UK, 2002, pp. 1-24.

[12] P. Comon, G. Golub, L.-H. Lim, and B. Mourrain, "Symmetric tensors and symmetric tensor rank," SIAM. J. Matrix Anal. Appl., vol. 30, no. 3, pp. 1254-1279, 2008.

[13] C. Cui, Y. Dai and J. Nie, "All real eigenvalues of symmetric tensors", SIAM. J. Matrix Anal. Appl. 35, pp. 1582-1601, 2014.

[14] L. de Lathauwer, B. de Moor, and J. Vandewalle, "A multilinear singular value decomposition," SIAM. J. Matrix Anal. Appl. 20, pp. 1253-1278, 2000.

[15] L. de Lathauwer, B. De Moor, and J. Vandewalle, "Computation of the canonical decomposition by means of a simultaneous generalized schur decomposition", SIAM. J. Matrix Anal. Appl. 26(2), 295-327, 2004.

[16] V. de. Silva and L.-H. Lim, "Tensor rank and the ill-posedness of the best low-rank approximation problem", SIAM. J. Matrix Anal. Appl., vol. 30, no. 3, pp. 1084-1127, 2008.

[17] W. Ding, L. Qi, and Y. Wei, "Inheritance properties and sum-of-squares decomposition of Hankel tensors: theory and algorithms", BIT 57, no. 1, pp. 169-190, 2017.

[18] I. Domanov and L. De Lathauwer, "Canonical polyadic decomposition of third-order tensors: reduction to generalized eigenvalue decomposition", SIAM J. Matrix Anal. Appl. 35(2), pp. 636-660, 2014.

[19] S. Friedland, "Remarks on the symmetric rank of symmetric tensors", SIAM. J. Matrix Anal. Appl., vol. 37, no. 1, pp. 320-337, 2016.

[20] S. Friedland and L.-H. Lim, "Nuclear norm of higher order tensors," Mathematics of Computation, to appear.

[21] F. Galuppi and M. Mella, "Identifiability of homogeneous polynomials and Cremona Transformations", Preprint, arXiv:1606.06895v2 [math.AG], 2016.

[22] L. Grasedyck, D. Kressner and C. Tobler, "A literature survey of lowrank tensor approximation techniques," Gammmitteilungen 36, no. 1, pp. 53-78, 2013.

[23] D. Grayson and M. Stillman, "Macaulay2: a software system for research in algebraic geometry", http://www.math.uiuc.edu/Macaulay2/

[24] J. Harris, Algebraic Geometry, A First Course, Graduate Texts in Mathematics, Springer, Berlin, 1992.

[25] J. Håstad, "Tensor rank is NP-complete," Journal of Algorithm 11(4), pp. 644-654, 1990.

[26] C. J. Hillar, L.-H. Lim, "Most tensor problems are NP-Hard," Journal of the ACM 60(6), article 45, 2013.

[27] F. L. Hitchcock, "The expression of a tensor or a polyadic as a sum of products," Journal of Mathematics and Physics 6 , pp. $164-189,1927$.

[28] A. Iarrobino and V. Kanev, Power Sums, Gorenstein algebras, and determinantal varieties. Lecture Notes in Mathematics \#1721, Springer, 1999.

[29] M. Ishteva, P.-A. Absil, S. Van Huffel and L. De Lathauwer, "Best Low Multilinear Rank Approximation of Higher-Order Tensors, Based on the Riemannian Trust-Region Scheme", SIAM. J. Matrix Anal. Appl. 32(1), pp. 115-135, 2011.

[30] T. Kolda and B. W. Bader, "Tensor decompositions and applications", SIAM Rev. 51(3), pp. 455-500, 2009.

[31] J. Kruskal, "Three-way arrays: rank and uniqueness of trilinear decompositions, with application to arithmetic complexity and statistics," Linear algebra and applications 18(2), pp. 95-138, 1977.

[32] J. Landsberg and L. Manivel, "On the ideals of secant varieties of Segre varieties", Found. Comput. Math. 4(4), pp. 397$422,2004$.

[33] J. Landsberg and J. Weyman, "On the ideals and singularities of secant varieties of Segre varieties", Bulletin of the London Mathematical Society 39, pp. 685-697, 2007.

[34] J. Landsberg and Z. Teitler, "On the ranks and border ranks of symmetric tensors," Found. Comput. Math. 10(3), pp. 339-366, 2010. 
[35] J. Landsberg. Tensors: geometry and applications. Graduate Studies in Mathematics, 128, American Mathematical Society, Providence, RI, 2012.

[36] J. Landsberg, "New lower bounds for the rank of matrix multiplication," SIAM J. Comput. 43(1), pp. 144-149, 2014.

[37] J. Landsberg and G. Ottaviani, "New lower bounds for the border rank of matrix multiplication", Theory of Computing, vol. 11 , pp. $285-298,2015$.

[38] S. Leurgans, R. T. Ross, and R. B. Abel, "A decomposition for three-way arrays", SIAM J. Matrix Anal. Appl. 14, pp. 1064-1083, 1993.

[39] L.-H. Lim, "Singular values and eigenvalues of tensors: a variational approach", Proceedings of IEEE International Workshop on Computational Advancs in Multi-Sensor Adaptive Processing (CAMSAP'05), 1, pp. 129-132, 2005.

[40] L.-H. Lim and P. Comon, "Multiarray signal processing: tensor decomposition meets compressed sensing," Comptes Rendus de l'Acadmie des Sciences - Series IIB - Mechanics 338(6), pp. 311-320, 2010.

[41] L.-H. Lim, Tensors and hypermatrices, in: L. Hogben (Ed.), Handbook of linear algebra, 2nd Ed., CRC Press, Boca Raton, FL, 2013.

[42] X. Liu and N. Sidiropoulos, "Almost Sure Identifiability of Constant Modulus Multidimen- sional Harmonic Retrieval," IEEE Transactions on Signal Processing 50, pp. 2366-2368, 2002.

[43] J. Nie and L. Wang, "Semidefinite relaxations for best rank-1 tensor approximations", SIAM. J. Matrix Anal. Appl. 35(3), pp. 1155-1179, 2014.

[44] J. Nie, "Generating polynomials and symmetric tensor decompositions", Found. Comput. Math. 17(2), pp. 423-465, 2017.

[45] J. Nie. Low rank symmetric tensor approximations. SIAM J. Matrix Anal. Appl., 38(4), pp. 1517-1540, 2017.

[46] J. Nie and X. Zhang, "Real eigenvalues of nonsymmetric tensors", Comp. Opt. and Appl. 70(1), pp. 1-32, 2018.

[47] L. Oeding, "Report on Geometry and representation theory of tensors for computer science, statistics and other areas", Preprint, arXiv:0810.3940 [math.AG], 2008.

[48] L. Oeding and G. Ottaviani, "Eigenvectors of tensors and algorithms for Waring decomposition", J. Symbolic Comput. 54, pp. 9-35, 2013.

[49] L. Oeding, "Border ranks of monomials", Preprint, arXiv:1608.02530 [math.AG], 2016.

[50] G. Ottaviani, "Symplectic bundles on the plane, secant varieties and Lüroth quartics revisited", Vector bundles and low codimensional subvarieties: state of the art and recent developments, 315-352, Quad. Mat. 21, Dept. Math., Seconda Univ. Napoli, Caserta, 2007.

[51] J. Papy, L. de Lathauwer, and S. Van Huffel, "Exponential data fitting using multilinear algebra. The single-channel and the multichannel case," Numerical Linear Algebra and Applications 12, pp. 809-826, 2005.

[52] L. Qi, "Eigenvalues of a real supersymmetric tensor", Journal of Symbolic Computation 40, pp. 1302-1324, 2005.

[53] L. Qi, "Eigenvalues and invariants of tensors", Journal of Mathematics Analysis and Applications 325, pp. 1363-1377, 2007.

[54] L. Qi, "Hankel Tensors: Associated Hankel Matrices and Vandermonde rank decomposition", Communications in mathematical sciences 13(1), p. 113-125, 2014.

[55] Y. Shitov, "How hard is the tensor rank?", Preprint, arXiv:1611.01559[math.C0], 2016.

[56] Y. Shitov, "A counterexample to Comon's conjecture," Preprint, arXiv:1705.08740[math.C0], 2017.

[57] J. Sylvester, "An essay on canonical forms, supplement to a sketch of a memoir on elimination, transformation and canonical forms," originally published by George Bell, Fleet Street, London, 1851; Paper 34 in Mathematical Papers (1973), vol. 1, Chelsea, New York. Originally published by Cambridge University Press in 1904.

[58] J. Sylvester, "On a remarkable discovery in the theory of canonical forms and of hyperdeterminants," originally in Phiosophical Magazine (1851), vol. 2; Paper 42 in Mathematical Papers (1973), vol. 1, Chelsea, New York. Originally published by Cambridge University Press in 1904.

[59] S. Trickett, L. Burroughs and A. Milton, "Interpolation using Hankel tensor completion," SEG Technical Program Expanded Abstracts, pp. 3634-3638, 2013.

[60] K. Ye and L.-H. Lim, "Fast structured matrix computations: tensor rank and cohn-umans method," Found. Comput. Math., issue 1, pp. 45-95, 2018.

[61] K. Ye and L.-H. Lim, "Tensor network states ranks", Preprint, arXiv:1801.02662 [math.NA], 2018.

\section{Appendix A. The proof of Lemma 5.3}

In this Appendix, we give the proof of Lemma 5.3 which is used in the proof of Theorem 5.2 We will also work out some examples to illustrate the idea of the proof. Readers are recommended to read these examples to better understand the proof. We first briefly describe the strategy we employ to prove Lemma 5.3 .

- first we investigate entries of $M$ to conclude that $M$ is a block diagonal matrix with blocks $M_{0}, \ldots, M_{p}$ where $M_{s}$ has $\left(\begin{array}{c}n-p-1 \\ s\end{array}\right)\left(\begin{array}{c}p \\ s-1\end{array}\right)(p+1)$ columns. This is done in Step 1-3 below;

- then we prove that each $M_{s}$ is of full rank by showing that the reduction of $M_{s}$ from $\mathbb{Z}$ to $\mathbb{Z}_{2}$ is non-singular. This is done in Step 4 and 5 below.

Proof of Lemma 5.3. By definition of $R$ and $C$, we have

$$
\# R=\left(\begin{array}{l}
n-1 \\
p-1
\end{array}\right) \geq \# C=\left(\begin{array}{l}
n-1 \\
p+1
\end{array}\right) .
$$


Then $D$ is a $\left(\begin{array}{c}n-1 \\ p\end{array}\right) n \times\left(\begin{array}{c}n-1 \\ p\end{array}\right) n$ matrix, $T_{1}$ is a $\left(\begin{array}{c}n-1 \\ p\end{array}\right) n \times\left(\begin{array}{c}n-1 \\ p+1\end{array}\right) n$ matrix and $T_{2}$ is a $\left(\begin{array}{c}n-1 \\ p-1\end{array}\right) n \times\left(\begin{array}{c}n-1 \\ p\end{array}\right) n$ matrix. This implies that $M=-T_{2} D T_{1}$ is a $\left(\begin{array}{c}n-1 \\ p-1\end{array}\right) n \times\left(\begin{array}{c}n-1 \\ p+1\end{array}\right) n$. For each $J^{\prime} \in C$ and $1 \leq k \leq n$, we denote by $v_{J^{\prime}, k}$ the $\left(J^{\prime}, k\right)$-th column vector of $M$. We will prove that the matrix $M$ has $\operatorname{rank}\left(\begin{array}{c}n-1 \\ p+1\end{array}\right)(p+1)$ in the following steps.

Step 1: We describe blocks $M_{J, J^{\prime}}$ of the matrix $M$. We may partition $T_{1}$ by blocks of size $n \times n$ and index them by elements in $R_{0}$ and $C$. To be more precise, for each $J \in R_{0}$ and $J^{\prime} \in C$ we denote by $T_{1, J, J^{\prime}}$ the submatrix obtained by taking rows $(J, 1), \cdots,(J, n)$ and columns $\left(J^{\prime}, 1\right), \ldots,\left(J^{\prime}, n\right)$. Similarly, we may also partition $T_{2}$ (resp. $D$ ) by blocks of size $n \times n$ and index them by elements in $R$ (resp. $R_{0}$ ) and $C_{0}$ (resp. $\left.C_{0}\right)$. We denote these blocks by $T_{2, J, J^{\prime}}, J \in R, J^{\prime} \in C_{0}\left(\operatorname{resp} . D_{J^{\prime}, J}, J^{\prime} \in C_{0}, J \in R_{0}\right)$. Since $M=-T_{2} D T_{1}$, we may partition $M$ in the same fashion and denote these blocks by $M_{J, J^{\prime}}, J \in R, J^{\prime} \in C$. Moreover,

$$
M_{J, J^{\prime}}=-\sum_{J_{0}^{\prime} \in C_{0}, J_{0} \in R_{0}} T_{2, J, J_{0}^{\prime}} D_{J_{0}^{\prime}, J_{0}} T_{1, J_{0}, J^{\prime}} .
$$

We notice that

- $D_{J_{0}^{\prime}, J_{0}} \neq 0$ if and only if $J_{0}^{\prime}=J_{0} \cup\{\lfloor(n+1) / 2\rfloor\}, J_{0}^{\prime} \in C_{0}, J_{0} \in R_{0}$.

- $T_{2, J, J_{0}^{\prime}} \neq 0$ only if $J \subsetneq J_{0}^{\prime}, J \in R, J_{0}^{\prime} \in C_{0}$.

- $T_{1, J_{0}, J^{\prime}} \neq 0$ only if $J_{0} \subsetneq J^{\prime}, J_{0} \in R_{0}, J^{\prime} \in C$.

Therefore, $M_{J, J^{\prime}} \neq 0$ only if there exists $j<\lfloor(n+1) / 2\rfloor<j^{\prime}$ such that

$$
J^{\prime}=J \backslash\{\lfloor(n+1) / 2\rfloor\} \cup\left\{j, j^{\prime}\right\},
$$

and

$$
\begin{aligned}
M_{J, J^{\prime}}= & -\left(T_{2, J, J \cup\{j\}} D_{J \cup\{j\}, J^{\prime} \backslash\left\{j^{\prime}\right\}} T_{1, J^{\prime} \backslash\left\{j^{\prime}\right\}, J^{\prime}}\right. \\
& \left.+T_{2, J, J \cup\left\{j^{\prime}\right\}} D_{J \cup\left\{j^{\prime}\right\}, J^{\prime} \backslash\{j\}} T_{1, J^{\prime} \backslash\{j\}, J^{\prime}}\right) .
\end{aligned}
$$

On the other hand, we recall from the proof of Theorem 5.2 that

$$
D_{J \cup\lfloor(n+1) / 2\rfloor, J}=\epsilon_{J, J \cup\{\lfloor(n+1) / 2\rfloor\}} \operatorname{Id}_{n}, \quad T_{s, J, J \cup\{j\}}=\epsilon_{J, J \cup\{j\}} T_{j-\lfloor(n+1) / 2\rfloor}, \quad s=1,2 .
$$

Here $T_{l}$ is the Toeplitz matrix $\left(t_{i j}\right)$ defined by

$$
t_{i j}= \begin{cases}1, & \text { if } j-i=l \\ 0, & \text { otherwise }\end{cases}
$$

Thus we obtain

$$
\begin{aligned}
T_{2, J, J \cup\{j\}} & =\epsilon_{J, J \cup\{j\}} T_{j-\lfloor(n+1) / 2\rfloor}, \\
D_{J \cup\{j\}, J^{\prime} \backslash\left\{j^{\prime}\right\}} & =\epsilon_{J^{\prime} \backslash\left\{j^{\prime}\right\}, J^{\prime} \backslash\left\{j^{\prime}\right\} \cup\{\lfloor(n+1) / 2\rfloor\}} \operatorname{Id}_{n}, \\
T_{1, J^{\prime} \backslash\left\{j^{\prime}\right\}, J^{\prime}} & =\epsilon_{J^{\prime} \backslash\left\{j^{\prime}\right\}, J^{\prime}} T_{j^{\prime}-\lfloor(n+1) / 2\rfloor}, \\
T_{2, J, J \cup\left\{j^{\prime}\right\}} & =\epsilon_{J, J \cup\left\{j^{\prime}\right\}} T_{j^{\prime}-\lfloor(n+1) / 2\rfloor}, \\
D_{J \cup\left\{j^{\prime}\right\}, J^{\prime} \backslash\{j\}} & =\epsilon_{J^{\prime} \backslash\{j\}, J^{\prime} \backslash\{j\} \cup\{\lfloor(n+1) / 2\rfloor\}} \operatorname{Id}_{n}, \\
T_{1, J^{\prime} \backslash\{j\}, J^{\prime}} & =\epsilon_{J^{\prime} \backslash\{j\}, J^{\prime}} T_{j-\lfloor(n+1) / 2\rfloor} .
\end{aligned}
$$

We set

$$
\begin{aligned}
& \delta_{J, J^{\prime}}=\delta_{J, j, j^{\prime}}=\epsilon_{J, J \cup\{j\}} \epsilon_{J^{\prime} \backslash\left\{j^{\prime}\right\}, J^{\prime} \backslash\left\{j^{\prime}\right\} \cup\{\lfloor(n+1) / 2\rfloor\}} \epsilon_{J^{\prime} \backslash\left\{j^{\prime}\right\}, J^{\prime}}
\end{aligned}
$$

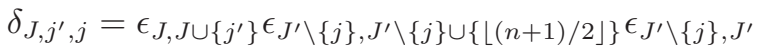

and it is straightforward to verify that $\delta_{J, j^{\prime}, j}=-\delta_{J, j, j^{\prime}}$. Hence we may write

$$
M_{J, J^{\prime}}=\delta_{J, J^{\prime}}\left(T_{j^{\prime}-\lfloor(n+1) / 2\rfloor} T_{j-\lfloor(n+1) / 2\rfloor}-T_{j-\lfloor(n+1) / 2\rfloor} T_{j^{\prime}-\lfloor(n+1) / 2\rfloor}\right),
$$

if $j<r-n+1<j^{\prime}$ and $J^{\prime}=J \backslash\{\lfloor(n+1) / 2\rfloor\} \cup\left\{j, j^{\prime}\right\}$ and $M_{J, J^{\prime}}=0$ otherwise.

Step 2: If $j<r-n+1<j^{\prime}$ and $J^{\prime}=J \backslash\{\lfloor(n+1) / 2\rfloor\} \cup\left\{j, j^{\prime}\right\}$, then by A.1 , the (i, $k$ )-th entry $\left(M_{J, J^{\prime}}\right)_{i, k}$ of the $n \times n$ matrix $M_{J, J^{\prime}}$ is zero unless $k-i=j+j^{\prime}-2\lfloor(n+1) / 2\rfloor$ and in this case, we have:

\footnotetext{
${ }^{8}$ Here we remark that according to the definition of $D$, we should denote each block by $D_{J, J^{\prime}}$ where $J \in R_{0}, J^{\prime} \in C_{0}$, but we switch $J$ with $J^{\prime}$ to simplify our notation. This is valid as $\# R_{0}=\# C_{0}$ and $D^{\top}=D$.
} 
- if $j+j^{\prime}-2\lfloor(n+1) / 2\rfloor \geq 0$, then

$$
\left(M_{J, J^{\prime}}\right)_{i, k}= \begin{cases}\delta_{J, J^{\prime}}, & \text { if } j^{\prime}-\lfloor(n+1) / 2\rfloor \geq k \geq j+j^{\prime}-2\lfloor(n+1) / 2\rfloor+1, \\ -\delta_{J, J^{\prime}}, & \text { if } n \geq k \geq 2 n-r+j, \\ 0, & \text { otherwise. }\end{cases}
$$

- if $j+j^{\prime}-2\lfloor(n+1) / 2\rfloor<0$, then

$$
\left(M_{J, J^{\prime}}\right)_{i, k}= \begin{cases}\delta_{J, J^{\prime}}, & \text { if } j^{\prime}-\lfloor(n+1) / 2\rfloor \geq k \geq 1, \\ -\delta_{J, J^{\prime}}, & \text { if } j+j^{\prime}-2 r+3 n-2 \geq k \geq j-r+2 n, \\ 0, & \text { otherwise. }\end{cases}
$$

In particular, we have

$$
\left(M_{J, J^{\prime}}\right)_{i,\left\lfloor\frac{n+1}{2}\right\rfloor}=\left(M_{J, J^{\prime}}\right)_{\left\lfloor\frac{n+1}{2}\right\rfloor, k}=0
$$

for any $J \in R, J^{\prime} \in C$ and $1 \leq i \leq n$. By (A.2) and (A.3), we see that $\left(M_{J, J^{\prime}}\right)_{i, k}=0$ unless

- $J^{\prime}=J \backslash\left\{\left\lfloor\frac{n+1}{2}\right\rfloor\right\} \cup\left\{j, j^{\prime}\right\}, j<\left\lfloor\frac{n+1}{2}\right\rfloor<j^{\prime}$,

- $k-i=\left(j+j^{\prime}\right)-2\left\lfloor\frac{n+1}{2}\right\rfloor$,

- if $j+j^{\prime}-2\left\lfloor\frac{n+1}{2}\right\rfloor \geq 0$, then

$$
k \in\left[j+j^{\prime}-2\left\lfloor\frac{n+1}{2}\right\rfloor+1, j^{\prime}-\left\lfloor\frac{n+1}{2}\right\rfloor\right] \cup[2 n-r+j, n],
$$

if $j+j^{\prime}-2\left\lfloor\frac{n+1}{2}\right\rfloor<0$, then

$$
k \in\left[1, j^{\prime}-\left\lfloor\frac{n+1}{2}\right\rfloor\right] \cup\left[j-r+2 n, j+j^{\prime}-2 r+3 n-2\right] .
$$

In particular, if both $\left(M_{J, J^{\prime}}\right)_{i, k}$ and $\left(M_{J, \widetilde{J}^{\prime}}\right)_{i, \widetilde{k}}$ are nonzero, then we must have

$$
\widetilde{k}-k=\sum_{t=1}^{p+1}\left(\widetilde{j_{t}^{\prime}}-j_{t}^{\prime}\right)=\left(\widetilde{j}+\widetilde{j^{\prime}}\right)-\left(j+j^{\prime}\right),
$$

where $J^{\prime}=\left(j_{1}^{\prime}<\cdots<j_{p+1}^{\prime}\right)=J \backslash\{\lfloor(n+1) / 2\rfloor\} \cup\left\{j, j^{\prime}\right\}$ and $\widetilde{J^{\prime}}=\left(\widetilde{j_{1}^{\prime}}<\cdots<\widetilde{j_{p+1}^{\prime}}\right)=J \backslash\{\lfloor(n+1) / 2\rfloor\} \cup$ $\left\{\widetilde{j}, \widetilde{j^{\prime}}\right\}$. Similarly, if both $\left(M_{J, J^{\prime}}\right)_{i, k}$ and $\left(M_{\widetilde{J}, J^{\prime}}\right)_{\widetilde{i}, k}$ are nonzero, then we must have

$$
\widetilde{i}-i=\left(\widetilde{j}+\widetilde{j^{\prime}}\right)-\left(j+j^{\prime}\right),
$$

where $J \backslash\lfloor(n+1) / 2\rfloor \cup\left\{j, j^{\prime}\right\}=J^{\prime}=\widetilde{J} \backslash\lfloor(n+1) / 2\rfloor \cup\left\{\widetilde{j}, \widetilde{j^{\prime}}\right\}$.

Step 3: We may write $J \in R$ as

$$
J=\left(j_{1}<\cdots<j_{s-1}<j_{s}=\lfloor(n+1) / 2\rfloor<j_{s+1}<\cdots<j_{p}\right)
$$

and write $J^{\prime} \in C$ as

$$
J^{\prime}=\left(j_{1}^{\prime}<\cdots<j_{t}^{\prime}<j_{t+1}^{\prime}<\cdots<j_{p+1}^{\prime}\right),
$$

where $j_{t}^{\prime}<\lfloor(n+1) / 2\rfloor<j_{t+1}^{\prime}$. By (A.1), we see that in particular, $M_{J, J^{\prime}}=0$ if $s+1 \neq t$. This implies that the matrix $M$ is a block diagonal matrix $M=\operatorname{Diag}\left\{M_{1}, \ldots, M_{p}\right\}$ where $M_{s}, 1 \leq s \leq\lfloor(n-1) / 2\rfloor$ is the submatrix of $M$ obtained by taking $J=\left(j_{1}<\cdots<j_{p}\right) \in R$ and $J^{\prime}=\left(j_{1}^{\prime}<\cdots<j_{p+1}^{\prime}\right) \in C$ such that

$$
j_{s}=\lfloor(n+1) / 2\rfloor, \quad j_{s+1}^{\prime}<\lfloor(n+1) / 2\rfloor<j_{s+2}^{\prime} .
$$

We define $R_{s}$ to be the subset of $R$ consisting of all $J=\left(j_{1}<\cdots<j_{p}\right) \in R$ such that $j_{s}=\lfloor(n+1) / 2\rfloor$ and define $C_{s}$ to be the subset of $C$ consisting of all $J^{\prime}=\left(j_{1}^{\prime}<\cdots<j_{p+1}^{\prime}\right) \in C$ such that $j_{s}^{\prime}<\lfloor(n+1) / 2\rfloor<j_{s+1}^{\prime}$.

For each $1 \leq s \leq\lfloor(n-1) / 2\rfloor$ and $J \in R_{s}, J^{\prime} \in C_{s}$, we remove $(J, i)$-th row from $M_{s}$ where $p-s+2 \leq$ $i \leq n-s$ and we remove $\left(J^{\prime}, k\right)$-th column from $M_{s}$ where $p-s+2 \leq k \leq n-s$. We still denote the new matrix by $M_{s}$. We denote by $v_{J^{\prime}, k}$ the $\left(J^{\prime}, k\right)$-th column vector of $M_{s}$ if $J^{\prime} \in C_{s}$. We use the same notation to denote column vectors of $M$ before, but since $M$ is a block diagonal matrix with diagonal blocks $M_{0}, \ldots, M_{p}$, this abuse of the notation should cause no confusion. We remark that the matrix $M_{s}$ is of size

$$
\left(\begin{array}{c}
n-p-1 \\
s-1
\end{array}\right)\left(\begin{array}{l}
p \\
s
\end{array}\right)(p+1) \times\left(\begin{array}{c}
n-p-1 \\
s
\end{array}\right)\left(\begin{array}{c}
p \\
s-1
\end{array}\right)(p+1)
$$


and thus it has more rows than columns. Hence it suffices to prove that the matrix $M_{s}$ has full rank, or equivalently, the set

$$
S_{s}=\left\{v_{J^{\prime}, k}: J^{\prime} \in C_{s}, k=1, \ldots, p-s+1, n-s+1, \ldots, n\right\}
$$

is a linearly independent set.

Step 4: Let $s$ be an integer such that $1 \leq s \leq\lfloor(n-1) / 2\rfloor$ and let $S_{s}$ be the set defined in (A.7). To prove that $S_{s}$ is a linearly independent set, we consider

$$
\sum_{\substack{J^{\prime} \in C_{s} \\ k=1, \ldots, p-s+1, n-s+1, \ldots, n}} x_{J^{\prime}, k} v_{J^{\prime}, k}=0,
$$

where $x_{J^{\prime}, k}$ 's are unknowns and we want to prove that $x_{J^{\prime}, k}=0$ for all $J^{\prime} \in C_{s}$ and $k=1, \ldots, p-s+1, n-$ $s+1, \ldots, n$. Since $v_{J^{\prime}, k}$ is the $\left(J^{\prime}, k\right)$-th column vector of $M_{s}$, the $(J, i)$-th entry $v_{J^{\prime}, k}^{J, i}$ of $v_{J^{\prime}, k}$ is equal to the $(i, k)$-th entry $\left(M_{J, J^{\prime}}\right)_{i, k}$ of $M_{J, J^{\prime}}$ defined by A.2 and A.3. Hence from wave for ach $J \in R_{s}$ and $1 \leq i \leq n$ the following linear equation:

$$
\sum_{\substack{J^{\prime} \in C_{s} \\ k=1, \ldots, p-s+1, n-s+1, \ldots, n}}\left(M_{J, J^{\prime}}\right)_{i, k} x_{J^{\prime}, k}=0 .
$$

We notice that if $\left(M_{J, J^{\prime}}\right)_{i, k} \neq 0$ and $k \leq p-s+1$ (resp. $\left.k \geq n-s+1\right)$, then whenever $\left(M_{J, \widetilde{J}^{\prime}}\right)_{i, \widetilde{k}} \neq 0$, we must also have $\widetilde{k} \leq p-s+1$ (resp. $\tilde{k} \geq n-s+1$ ). This can be seen from (A.5) and (A.4). Hence (A.9) can be simplified as:

$$
\begin{aligned}
\sum_{\substack{J^{\prime} \in C_{s} \\
k=1, \ldots, p-s+1}}\left(M_{J, J^{\prime}}\right)_{i, k} x_{J^{\prime}, k}=0 \quad \text { or } \\
\sum_{\substack{J^{\prime} \in C_{s} \\
k=n-s+1, \ldots, n}}\left(M_{J, J^{\prime}}\right)_{i, k} x_{J^{\prime}, k}=0
\end{aligned}
$$

We denote by $X_{J}^{i}$ the set of variables $x_{J^{\prime}, k}$ whose coefficient $\left(M_{J, J^{\prime}}\right)_{i, k} \neq 0$. Let $A=\left\{\left(J_{1}, i_{1}\right), \ldots,\left(J_{m}, i_{m}\right)\right\}$ be a maximal set such that for any $\left(J_{a}, i_{a}\right) \in A$, there exists some $\left(J_{b}, i_{b}\right) \in A, a \neq b$,

$$
X_{J_{a}}^{i_{a}} \cap X_{J_{b}}^{i_{b}} \neq \emptyset \text {. }
$$

If $x_{J_{0}^{\prime}, k_{0}} \notin \cup_{(J, i) \in A} X_{J}^{i}$ then we see that $x_{J_{0}^{\prime}, k_{0}}$ is independent on $x_{J^{\prime}, k} \in \cup_{(J, i) \in A} X_{J}^{i}$. Therefore, it is sufficient to prove that the solution of the linear system:

$$
\sum_{x_{J^{\prime}, k} \in X_{J_{t}}^{i_{t}}}\left(M_{J_{t}, J^{\prime}}\right)_{i_{t}, k} x_{J^{\prime}, k}=0, \quad t=1, \ldots, m .
$$

is zero. By A.10 and A.11, we see that

- either $k \leq p-s+1$ for all $x_{J^{\prime}, k} \in \cup_{(J, i) \in A} X_{J}^{i}$,

- or $k \geq n-s+1$ for all $x_{J^{\prime}, k} \in \cup_{(J, i) \in A} X_{J}^{i}$.

Similarly, we also have

- either $i \leq\lfloor p-s+1$ for all $(J, i) \in A$,

- or $i \geq n-s+1$ for all $(J, i) \in A$.

If there exists $x_{J^{\prime}, k} \in \cup_{(J, i) \in A} X_{J}^{i}$ such that $k \geq n-s+1$ and $i \leq p-s+1$, then $k-i \geq n-p$. However, we have

this implies that

$$
k-i=\left(j+j^{\prime}\right)-2\left\lfloor\frac{n+1}{2}\right\rfloor
$$

$$
j+j^{\prime} \geq n+2\left\lfloor\frac{n+1}{2}\right\rfloor-p \geq n+\left\lfloor\frac{n+1}{2}\right\rfloor,
$$

which contradicts the assumption that $j<\lfloor(n+1) / 2\rfloor<j^{\prime} \leq n$. Similarly, we may prove that if $k \leq p-s+1$ and $i \geq n-s+1$, then

$$
j+j^{\prime} \leq p-n+2\left\lfloor\frac{n+1}{2}\right\rfloor \leq p+1 \leq\left\lfloor\frac{n+1}{2}\right\rfloor+1
$$


which contradicts the assumption that $1 \leq j<\lfloor(n+1) / 2\rfloor<j^{\prime}$. Hence if $(J, i) \in A$ and $i \leq p-s+1$ (resp. $i \geq n-s+1$ ), then all $x_{J^{\prime}, k} \in \cup_{(J, i) \in A} X_{J}^{i}$ must have $k \leq p-s+1$ (resp. $k \geq n-s+1$ ). We denote by $E_{1}$ the set of integers $1,2, \ldots, p-s+1$ and by $E_{2}$ the set of integers $n-s+1, n-s+2, \ldots, n$.

According to (A.6) and (A.5), we may describe the set $A$ as follows: if $(J, i) \in A, i \in E_{s}, s=1,2$, then $(\widetilde{J}, \widetilde{i}) \in A$ if and only if

$$
\widetilde{i}-i=\sum_{t=1}^{p}\left(\widetilde{j_{t}}-j_{t}\right), \quad \widetilde{i} \in E_{s} .
$$

The set $\cup_{(J, i) \in A} X_{J}^{i}$ can be described as in a similar way: if $x_{J^{\prime}, k} \in \cup_{(J, i) \in A} X_{J}^{i}, i, k \in E_{s}, s=1,2$, then $x_{\widetilde{J}, \widetilde{k}} \in \cup_{(J, i) \in A} X_{J}^{i}$ if and only if

$$
\widetilde{k}-k=\sum_{t=1}^{p+1}\left(\widetilde{j_{t}^{\prime}}-j_{t}^{\prime}\right), \quad \widetilde{k} \in E_{s} .
$$

Step 5: $A=\left\{\left(J_{1}, i_{1}\right), \ldots,\left(J_{m}, i_{m}\right)\right\}$ be a maximal set such that for any $\left(J_{a}, i_{a}\right) \in A$ there exists some $\left(J_{b}, i_{b}\right) \in A$ such that

$$
X_{J_{a}}^{i_{a}} \cap X_{J_{b}}^{i_{b}} \neq \emptyset, \quad a \neq b .
$$

We prove that every $x_{J^{\prime}, k} \in \cup_{(J, i) \in A} X_{J}^{i}$ is equal to zero. To see this, it is sufficient to prove that the solution to the linear system (A.12) over the field $\mathbb{Z}_{2}=\{0,1\}$ must be trivial, i.e., $x_{J^{\prime}, k}=0$. Indeed, if (A.12) has a nontrivial solution over $\mathbb{C}$, then it also has a nontrivial solution over $\mathbb{Z}$ since coefficients of (A.12) are $-1,1$ or 0 and hence in particular are integers. Moreover, among these nontrivial integer solutions, there must be a solution $\left(a_{J^{\prime}, k}\right)_{x_{J^{\prime}, k} \in \cup_{(J, i) \in A} X_{J}^{i}}$ such that

$$
a_{J^{\prime}, k} \equiv 1 \quad(\bmod 2)
$$

for some $\left(J^{\prime}, k\right)$. Equivalently, A.12 has a nontrivial solution over $\mathbb{Z}_{2}$.

Step 6: We denote by $M_{s, A}$ the coefficient matrix of the system A.12 and we suppose that $M_{s, A}$ is an $m \times l$ matrix. By the construction of $M_{s, A}$ we know that $m \geq l$. We define an order on column indices $\left\{\left(J_{1}^{\prime}, k_{1}\right), \ldots,\left(J_{l}^{\prime}, k_{l}\right)\right\}$ of $M_{s, A}$ by $\left(J_{a}^{\prime}, k_{a}\right)>\left(J_{b}^{\prime}, k_{b}\right)$ if

$$
\sum_{t=s+1}^{p+1} j_{a t}^{\prime}-\sum_{t=1}^{s} j_{a t}^{\prime}>\sum_{t=s+1}^{p+1} j_{b t}^{\prime}-\sum_{t=1}^{s} j_{b t}^{\prime}
$$

or

$$
\sum_{t=s+1}^{p+1} j_{a t}^{\prime}-\sum_{t=1}^{s} j_{a t}^{\prime}=\sum_{t=s+1}^{p+1} j_{b t}^{\prime}-\sum_{t=1}^{s} j_{b t}^{\prime} \text { and } k_{a}>k_{b},
$$

where

$$
\begin{aligned}
J_{a}^{\prime} & =\left(j_{a, 1}^{\prime}<\cdots j_{a, s}^{\prime}<j_{a, s+1}^{\prime}<\cdots<j_{a, p+1}^{\prime}\right) \in C_{s}, \\
J_{b}^{\prime} & =\left(j_{b, 1}^{\prime}<\cdots j_{b, s}^{\prime}<j_{b, s+1}^{\prime}<\cdots<j_{b, p+1}^{\prime}\right) \in C_{s} .
\end{aligned}
$$

Similarly, we may define an order on the set $A=\left\{\left(J_{1}, i_{1}\right), \ldots,\left(J_{m}, i_{m}\right)\right\}$ by $\left(J_{a}, i_{a}\right)>\left(J_{b}, i_{b}\right)$ if

$$
\sum_{t=s+1}^{p} j_{a t}-\sum_{t=1}^{s-1} j_{a t}>\sum_{t=s+1}^{p} j_{b t}-\sum_{t=1}^{s-1} j_{b t}
$$

or

$$
\sum_{t=s+1}^{p} j_{a t}-\sum_{t=1}^{s-1} j_{a t}=\sum_{t=s+1}^{p} j_{b t}-\sum_{t=1}^{s-1} j_{b t} \text { and } i_{a}>i_{b}
$$

where

$$
\begin{aligned}
J_{a} & =\left(j_{a, 1}<\cdots<j_{a, s-1}<j_{a, s}=\lfloor(n+1) / 2\rfloor<j_{a, s+1}<\cdots<j_{a, p}\right) \in R_{s}, \\
J_{b} & =\left(j_{b, 1}<\cdots<j_{b, s-1}<j_{b, s}=\lfloor(n+1) / 2\rfloor<j_{b, s+1}<\cdots<j_{b, p}\right) \in R_{s} .
\end{aligned}
$$


With the order defined above, we may reorder $\left(J_{1}, i_{1}\right), \ldots,\left(J_{m}, i_{m}\right)$ and $\left(J_{1}^{\prime}, k_{1}\right), \ldots,\left(J_{l}^{\prime}, k_{l}\right)$ respectively so that

$$
\begin{gathered}
\left(J_{a}, i_{a}\right) \leq\left(J_{b}, i_{b}\right), \quad 1 \leq a<b \leq m, \\
\left(J_{\alpha}^{\prime}, k_{\alpha}\right) \leq\left(J_{\beta}^{\prime}, k_{\beta}\right), \quad 1 \leq \alpha<\beta \leq l .
\end{gathered}
$$

To prove that A.12) only has a trivial solution over $\mathbb{Z}_{2}$, it suffices to prove that

- there exists $(J, i) \in A$ such that the $(J, i)$-th row vector $w$ of $M_{s, A}$ has exactly one nonzero entry indexed by $\left(J^{\prime}, k\right)$ and

- The submatrix obtained by removing $(J, i)$-th row and $\left(J^{\prime}, k\right)$-th column from $M_{s, A}$ has full rank.

In fact, the row vector $w$ is one of the following:

- $w$ is the $\left(J_{1}, i_{1}\right)$-th row of $M_{s, A}$.

- $w$ is the $\left(J_{m}, i_{m}\right)$-th row of $M_{s, A}$.

- $w$ is the $(J, i)$-th row of $M_{s, A}$, where $(J, i)$ is maximal among those such that $\left(M_{s, A}\right)_{(J, i),\left(J_{1}^{\prime}, k_{1}\right)}$.

- $w$ is the $(J, i)$-th row of $M_{s, A}$, where $(J, i)$ is minimal among those such that $\left(M_{s, A}\right)_{(J, i),\left(J_{1}^{\prime}, k_{1}\right)}$.

- $w$ is the $(J, i)$-th row of $M_{s, A}$, where $(J, i)$ is maximal among those such that $\left(M_{s, A}\right)(J, i),\left(J_{l}^{\prime}, k_{l}\right)$.

- $w$ is the $(J, i)$-th row of $M_{s, A}$, where $(J, i)$ is minimal among those such that $\left(M_{s, A}\right)_{(J, i),\left(J_{l}^{\prime}, k_{l}\right)}$.

We denote by $M_{s, A}^{1}$ the matrix obtained by removing the $(J, i)$-th row and $\left(J^{\prime}, k\right)$-th column from $M_{s, A}$. Then $M_{s, A}^{1}$ has a row vector which contains exactly one nonzero entry. Indeed, we can find this row vector in the same way as we find the row vector $w$ for $M_{s, A}$. Hence by induction, we see that $M_{s, A}$ must have full rank and this completes the proof.

We illustrate the proof of Lemma 5.3 by some examples.

Example A.1. By the definition of $M_{s, A}$, we see that for any positive integer $n$, if $s=1$ or $\lfloor(n-1) / 2\rfloor$, then $M_{s, A}$ is simply a $p \times p$ triangular matrix whose diagonal entries are all 1 . In this case, we see that $M_{s, A}$ obviously has full rank.

Example A.2. Let $n=6$ and $s=2$, then $p=\left\lfloor\frac{n}{2}\right\rfloor=3$,

$$
\left\lfloor\frac{n+1}{2}\right\rfloor=3, \quad p+1-s=2, \quad n-s+1=5 .
$$

We also have

$$
\begin{aligned}
& C_{s}=\{(1,2,4,5),(1,2,4,6),(1,2,5,6)\}, \\
& R_{s}=\{(1,3,4),(1,3,5),(1,3,6),(2,3,4),(2,3,5),(2,3,6)\} .
\end{aligned}
$$

The matrix $M_{s}$ is

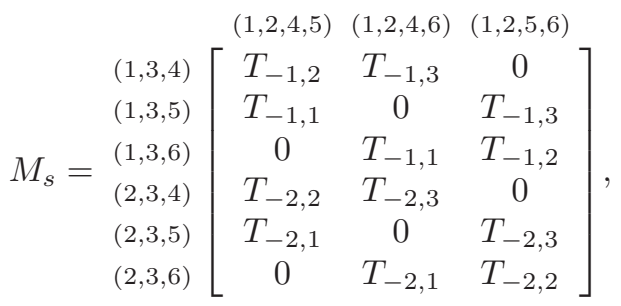

where $T_{a, b}$ is the submatrix obtained by removing columns $k=3,4$ and rows $i=3,4$ from $T_{a} T_{b}-T_{b} T_{a}$ over $\mathbb{Z}_{2}$. Here $T_{a}$ is the $n \times n$ Toeplitz matrix whose $(u, v)$-th entry is one if $v-u=a$ and is zero otherwise. For example, $T_{-1,2}$ and $T_{-2,2}$ are

$$
T_{-1,2}=\left[\begin{array}{llll}
0 & 1 & 0 & 0 \\
0 & 0 & 0 & 1 \\
0 & 0 & 0 & 0 \\
0 & 0 & 0 & 0
\end{array}\right], \quad T_{-2,2}=\left[\begin{array}{llll}
1 & 0 & 0 & 0 \\
0 & 1 & 0 & 0 \\
0 & 0 & 1 & 0 \\
0 & 0 & 0 & 1
\end{array}\right] .
$$

If we take

$$
A=\{((2,3,4), 1),((2,3,5), 2),((1,3,5), 1),((1,3,6), 2)\}
$$


then the matrix $M_{s, A}$ is

$$
M_{s, A}=\begin{array}{cc}
((2,3,4), 1) \\
((2,3,5), 2) \\
((1,3,5), 1)
\end{array}\left[\begin{array}{cc}
((1,2,4,5), 1) & ((1,2,4,6), 2) \\
1 & 1 \\
1 & 0 \\
1 & 0 \\
0 & 0
\end{array}\right] .
$$

It is straightforward to verify that the $((1,3,5), 1)$-th row of $M_{s, A}$ has only one nonzero entry, which is indexed by $((1,2,4,5), 1)$. We delete the $((1,3,5), 1)$-th row and the $((1,2,4,5), 1)$-th column of $M_{s, A}$ to obtain $M_{s, A}^{1}=[1]$, which has full rank.

Example A.3. We let $n=7, s=2$ then $p=3$,

$$
\left\lfloor\frac{n+1}{2}\right\rfloor=4, \quad p+1-s=2, \quad n-s+1=6 .
$$

We take

$$
A=\{((2,4,5), 1),((3,4,5), 2),((1,4,6), 1),((2,4,6), 2),((1,4,7), 2)\} .
$$

The matrix $M_{s, A}$ is

$$
\begin{gathered}
((3,4,5), 2) \\
((2,4,5), 1) \\
M_{s, A}= \\
((2,4,6), 2) \\
((1,4,6), 1) \\
((1,4,7), 2)
\end{gathered}\left[\begin{array}{ccccc}
((2,3,5,6), 2) & ((1,3,5,6), 1) & ((1,3,5,7), 2) & ((1,2,5,7), 1) & ((1,2,6,7), 2) \\
1 & 1 & 1 & 0 & 0 \\
1 & 0 & 0 & 1 & 0 \\
0 & 0 & 0 & 0 & 1 \\
0 & 1 & 0 & 0 & 1 \\
0 & 0 & 0 & 1 & 1
\end{array}\right] .
$$

We see that the $((2,4,6), 2)$-th row has the unique nonzero entry indexed by $((1,2,6,7), 2)$. We obtain $M_{s, A}^{1}$ by removing $((2,4,6), 2)$-th row and $((1,2,6,7), 2)$-th column:

$$
M_{s, A}^{1}=\begin{gathered}
((3,4,5), 2) \\
((2,4,5), 1) \\
((1,4,6), 1)
\end{gathered}\left(\begin{array}{cccc}
((2,4,3,5,6), 2) \\
1 & 1 & 1 & 0 \\
1 & 0 & 0 & 1 \\
0 & 1 & 0 & 0 \\
0 & 0 & 0 & 1
\end{array}\right] .
$$

By the same procedure, we obtain

$$
M_{s, A}^{2}=\underset{(((2,4,5), 2)}{((1,4,6), 1)}\left[\begin{array}{ccc}
((2,3,5,6), 2) & ((1,3,5,6), 1) & ((1,3,5,7), 2)) \\
1 & 1 & 1 \\
1 & 0 & 0 \\
0 & 1 & 0
\end{array}\right]
$$

by removing $((1,4,7), 1)$-th row and $((1,2,5,7), 1)$-th column of $M_{s, A}^{1}$ and we obtain

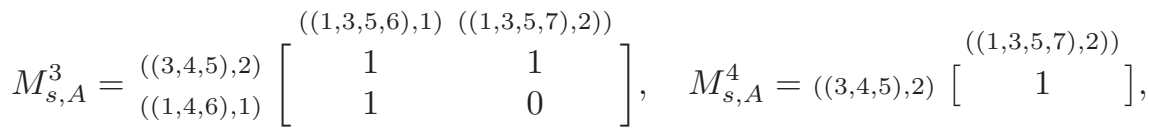

by removing $((2,4,5), 1)$-th row and $((2,3,5,6), 2)$-th column of $M_{s, A}^{2}$ and removing $((1,4,6), 1)$-th row and $((1,3,5,6), 1)$-th column of $M_{s, A}^{3}$, respectively.

Example A.4. Let $n=8, s=2$ then $p=\lfloor n / 2\rfloor=4$ and

$$
\left\lfloor\frac{n+1}{2}\right\rfloor=4, \quad p+1-s=3, \quad n-s+1=7 .
$$

We consider

$$
\begin{aligned}
& A=\{((3,4,5,6), 1),((3,4,5,7), 2),((3,4,6,7), 3),((2,4,5,7), 1),((3,4,5,8), 3), \\
&((2,4,5,8), 2),((1,4,6,7), 1),((2,4,6,8), 3),((1,4,6,8), 2),((1,4,7,8), 3)\} .
\end{aligned}
$$


The corresponding $M_{s, A}$ is

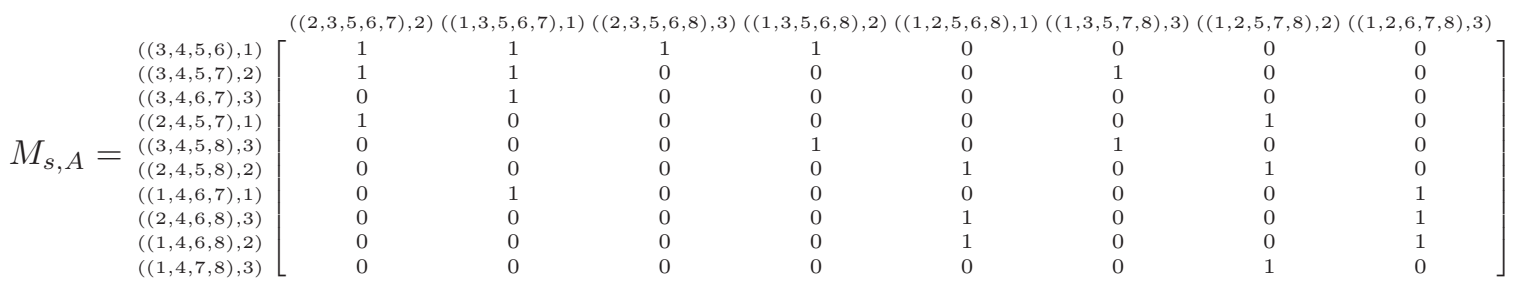

and we remove $((1,4,7,8), 3)$-th row and $((1,2,5,7,8), 2)$-th column of $M_{s, A}$ to obtain

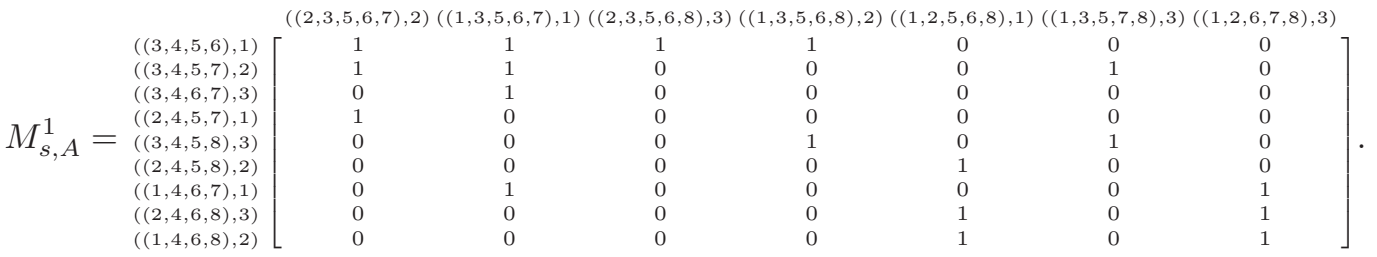

We remove $((2,4,5,7), 1)$-th row and $((2,3,5,6,7), 2)$-th column of $M_{s, A}^{1}$ to obtain

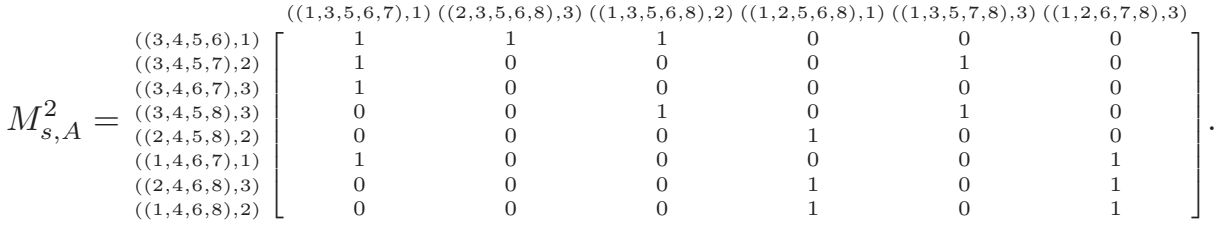

We remove $((3,4,6,7), 3)$-th row and $((1,3,5,6,7), 1)$-th column of $M_{s, A}^{3}$ to obtain

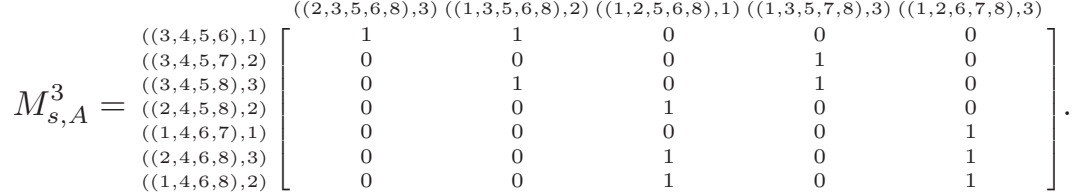

We remove $((1,4,6,7), 1)$-th row and $((1,2,6,7,8), 3)$-th column of $M_{s, A}^{3}$ to obtain

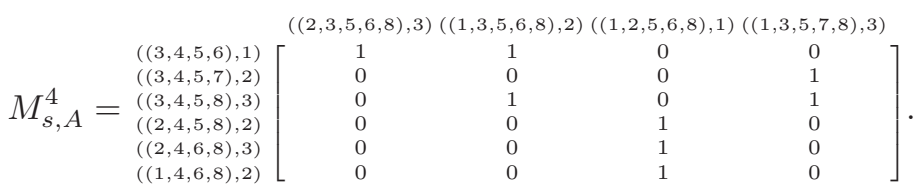

It is easy to determine $M_{s, A}^{5}, M_{s, A}^{6}$ and lastly $M_{s, A}^{7}=\left[\begin{array}{lll}1 & 0 & 0\end{array}\right]^{\top}$.

Department of Mathematics, University of California San Diego, 9500 Gilman Drive La Jolla, CA 92093

E-mail address: njw@math.ucsd.edu

Academy of Mathematics and System Sciences, Chinese Academy of Science, Beijing 100190, China

E-mail address: keyk@amss.ac.cn 